\title{
The Lunar Reconnaissance Orbiter Diviner Lunar Radiometer Experiment
}

\author{
D.A. Paige • M.C. Foote $\cdot$ B.T. Greenhagen $\cdot$ J.T. Schofield $\cdot$ S. Calcutt $\cdot$ \\ A.R. Vasavada - D.J. Preston - F.W. Taylor · C.C. Allen · K.J. Snook · B.M. Jakosky • \\ B.C. Murray · L.A. Soderblom • B. Jau · S. Loring · J. Bulharowski · N.E. Bowles • \\ I.R. Thomas · M.T. Sullivan · C. Avis · E.M. De Jong • W. Hartford • D.J. McCleese
}

Received: 24 January 2009 / Accepted: 7 May 2009 / Published online: 26 June 2009

(C) The author(s) 2009. This article is published with open access at Springerlink.com

\begin{abstract}
The Diviner Lunar Radiometer Experiment on NASA's Lunar Reconnaissance Orbiter will be the first instrument to systematically map the global thermal state of the Moon and its diurnal and seasonal variability. Diviner will measure reflected solar and emitted infrared radiation in nine spectral channels with wavelengths ranging from 0.3 to 400 microns. The resulting measurements will enable characterization of the lunar thermal environment, mapping surface properties such as thermal inertia, rock abundance and silicate mineralogy, and determination of the locations and temperatures of volatile cold traps in the lunar polar regions.
\end{abstract}

D.A. Paige $(\bowtie) \cdot$ B.T. Greenhagen · M.T. Sullivan

Dept. of Earth and Space Sciences, University of California, Los Angeles, USA

e-mail:dap@moon.ucla.edu

M.C. Foote - J.T. Schofield - A.R. Vasavada - D.J. Preston - B. Jau · S. Loring · J. Bulharowski

C. Avis · E.M. De Jong · W. Hartford - D.J. McCleese

Jet Propulsion Laboratory, California Institute of Technology, Pasadena, USA

S. Calcutt $\cdot$ F.W. Taylor $\cdot$ N.E. Bowles $\cdot$ I.R. Thomas

Dept. of Physics, University of Oxford, Oxford, UK

C.C. Allen

Lyndon B. Johnson Space Center, Houston, USA

K.J. Snook

NASA Headquarters, Washington DC, USA

B.M. Jakosky

Dept. of Geological Sciences, University of Colorado, Boulder, USA

B.C. Murray

Div. of Geological and Planetary Sciences, California Institute of Technology, Pasadena, USA

L.A. Soderblom

US Geologic Survey, Flagstaff, USA 
Keywords Moon · Lunar · Diviner · Thermal · Radiometer · Mapping · Temperature · Infrared $\cdot$ Mineralogy $\cdot$ Petrology $\cdot$ LRO $\cdot$ Reconnaissance Orbiter

\section{Introduction}

The Moon's surface thermal environment is among the most extreme of any planetary body in the solar system. Lunar temperatures at the subsolar point approach $400 \mathrm{~K}$, whereas temperatures in permanent shadow may be lower than $40 \mathrm{~K}$. The surface temperature of the Moon also represents a fundamental boundary condition that governs the thermal state of the Moon's regolith and interior, and the behavior of near-surface volatiles. The lunar thermal environment is also a significant challenge for human and robotic exploration systems, which must be designed to handle its extremes.

This paper describes the Diviner Lunar Radiometer Experiment, which is one of seven instruments aboard NASA's Lunar Reconnaissance Orbiter (LRO) mission. Diviner will be the first experiment to systematically map the global thermal state of the Moon and its diurnal and seasonal variability. In the sections below, we will describe the goals, instrumentation, observational strategy, and anticipated data products of the Diviner investigation.

\subsection{The Lunar Thermal Environment}

Observations and models show that the Moon has three distinct thermal environments: daytime, nighttime and polar. The Moon's daytime thermal environment is controlled by the flux of solar radiation. Because of the low thermal conductivity of the lunar soil, and the length of the lunar day, the temperatures of illuminated surfaces on the Moon are always close to radiative equilibrium. As illustrated in Fig. 1, the latitudinal and diurnal variation in lunar daytime temperatures is controlled mostly by the angular distance to the subsolar

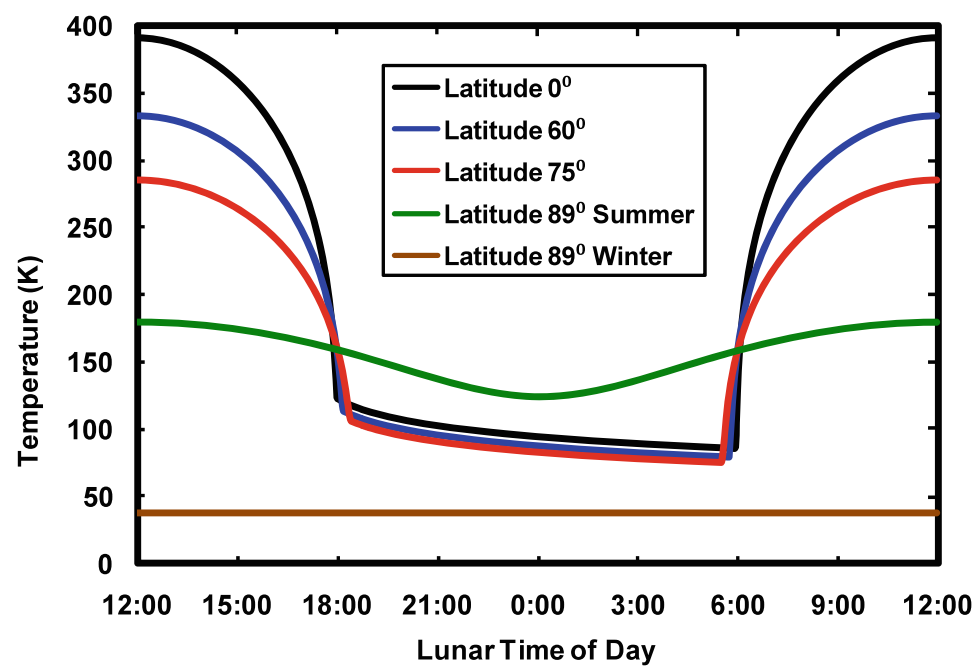

Fig. 1 Model calculations of lunar surface temperature variations as a function of local time and latitude. (Vasavada et al. 1999). Local time is expressed in lunar hours which correspond to $1 / 24$ of a lunar month. At $89^{\circ}$ latitude, diurnal temperature variations are shown at summer and winter solstices 


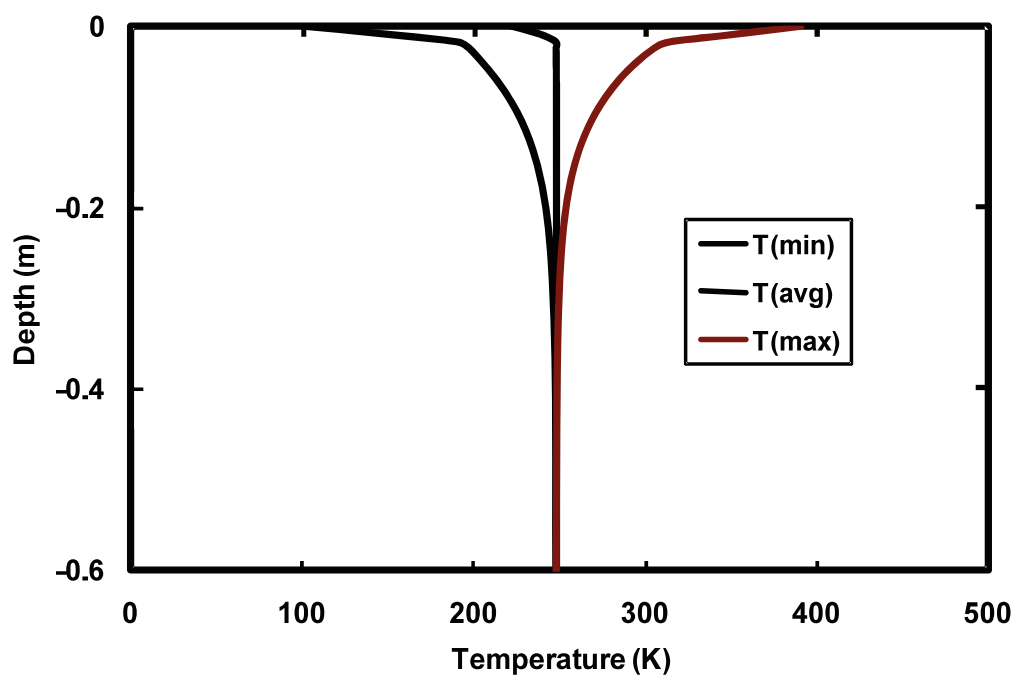

Fig. 2 Model calculations of diurnal minimum, maximum and average temperatures at the lunar equator as a function of depth (Vasavada et al. 1999)

point. The lunar nighttime environment is characterized by extreme cold. With no oceans or appreciable atmosphere to buffer surface temperatures, the only heat source to balance the loss of thermal radiation to space during the long lunar night is the sensible heat stored in the lunar subsurface during the day. The low thermal conductivity of the uppermost layers of the lunar regolith impedes the flow of heat from the subsurface to the surface, and as a consequence, lunar nighttime temperatures hover near $100 \mathrm{~K}$ for the duration of the 14-day lunar night. As illustrated in Fig. 2, the depth of penetration of the diurnal temperature wave on the Moon is $\sim 30 \mathrm{~cm}$.

The Moon also experiences seasonal insolation variations due to the combined effects of the $5.14^{\circ}$ obliquity of the Moon's orbital plane relative to the ecliptic, and the $6.68^{\circ}$ obliquity of the Moon's spin axis relative to the Moon's orbital plane. The net effect is that the latitude of the subsolar point undergoes a seasonal variation with an amplitude of $\sim 1.54^{\circ}$ and a period of $\sim 346$ days, which is less than a full Earth year due to the precession of the Moon's orbital plane. As a consequence, the polar regions experience very little direct solar illumination, and the illumination levels are strongly influenced by the effects of topography, as well as the lunar seasons. As illustrated in Fig. 1, a hypothetical horizontal surface at $89^{\circ}$ latitude would have temperatures ranging from 128 to $180 \mathrm{~K}$ during peak continuous illumination conditions at summer solstice, and temperatures near $38 \mathrm{~K}$ during un-illuminated polar night conditions at winter solstice. The depth of penetration of seasonal temperature waves is roughly square root of the ratio of the annual to diurnal insolation periods, or $\sim 120 \mathrm{~cm}$ at the lunar poles.

Superposed on the large-scale trends due to latitude, time of day, and season, the surface temperature of the Moon also exhibits variations due to topographic relief (slopes, roughness, shadows, and scattered solar and infrared radiation), spatial variations in solar reflectance and infrared emissivity, and spatial variations in the thermal properties of lunar surface materials (shown in Fig. 3). Because of the low thermal conductivity of the bulk of the lunar regolith, the lack of an appreciable atmosphere, and the effects of slopes and shadowing, the surface temperature of the Moon can exhibit extreme spatial variations all the 


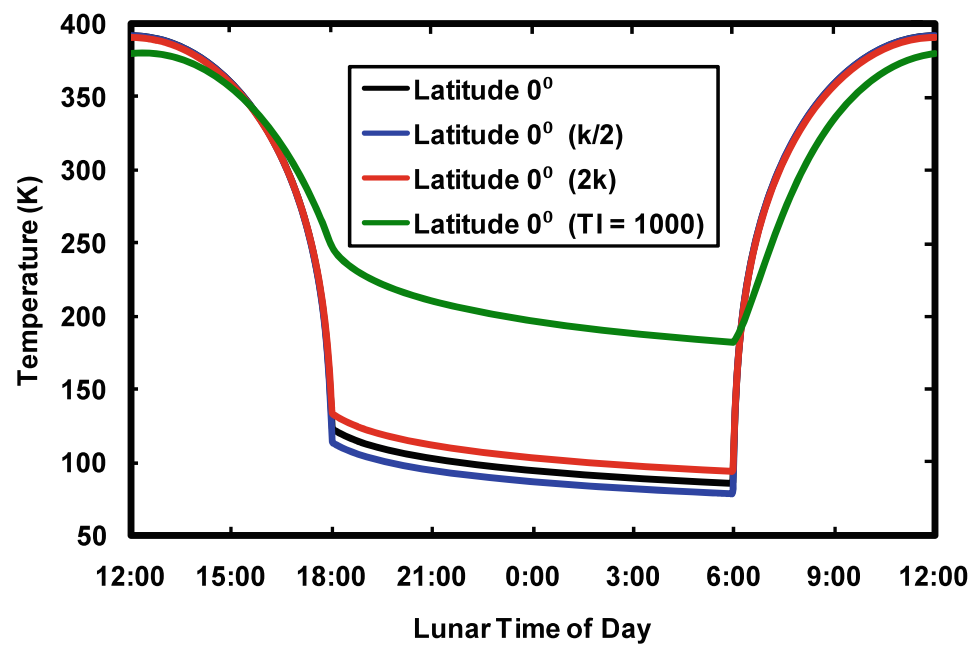

Fig. 3 Model calculations of the effects of regolith thermal properties and rocks on diurnal temperature variations at the equator (Vasavada et al. 1999). Shown are the effects of halving or doubling the thermal conductivity of the regolith, and those anticipated for a semi-infinite rock surface with a thermal inertia of $1000 \mathrm{~J} \mathrm{~m}^{-2} \mathrm{k}^{-1} \mathrm{~s}^{-1 / 2}$

way down to the $\sim 10 \mathrm{~cm}$ length scale of diurnal thermal skin depth. This fact significantly complicates the interpretation of lunar thermal observations and thermal model results, and the application of the information they provide to the design of practical lunar exploration systems.

Of special interest is the thermal behavior in high latitude regions that do not receive direct solar illumination. Thermal models that include the effects of multiply scattered solar and infrared radiation show that daily maximum surface temperatures in the coldest parts of larger permanently shadowed polar craters range from 40 to $90 \mathrm{~K}$, depending on latitude and crater geometry (Vasavada et al. 1999). Even lower temperatures are possible in smallerscale topographic features within permanently shadowed regions that are shaded from firstbounce indirect sunlight and/or infrared emission from warmer surfaces (Hodges 1980). The surface and subsurface thermal behavior in these coldest regions places fundamental constraints on the stability of cold-trapped water ice and other volatiles that may be coldtrapped at the lunar poles (Watson et al. 1961).

\subsection{Past Ground-Based and Spacecraft Measurements}

The first radiometric measurements of the temperature of the lunar surface were made by placing two uncooled thermopiles side by side at the focus of a reflecting telescope, one receiving radiation from the Moon and the other from space. Refinements of this original technique led to measurements of diurnal surface temperature variations over most of a lunation period, and during lunar eclipses (Petit and Nicholson 1930). Thermal modeling results showed that observed nighttime and eclipse temperatures were consistent with a $\sim 2 \mathrm{~cm}$-thick lunar surface layer composed of fine-grained low thermal conductivity regolith overlying material of higher thermal conductivity below (Jaeger and Harper 1950). More sophisticated ground-based infrared observations revealed the existence of spatial variations in nighttime brightness temperatures associated with bright rayed craters, which suggested 


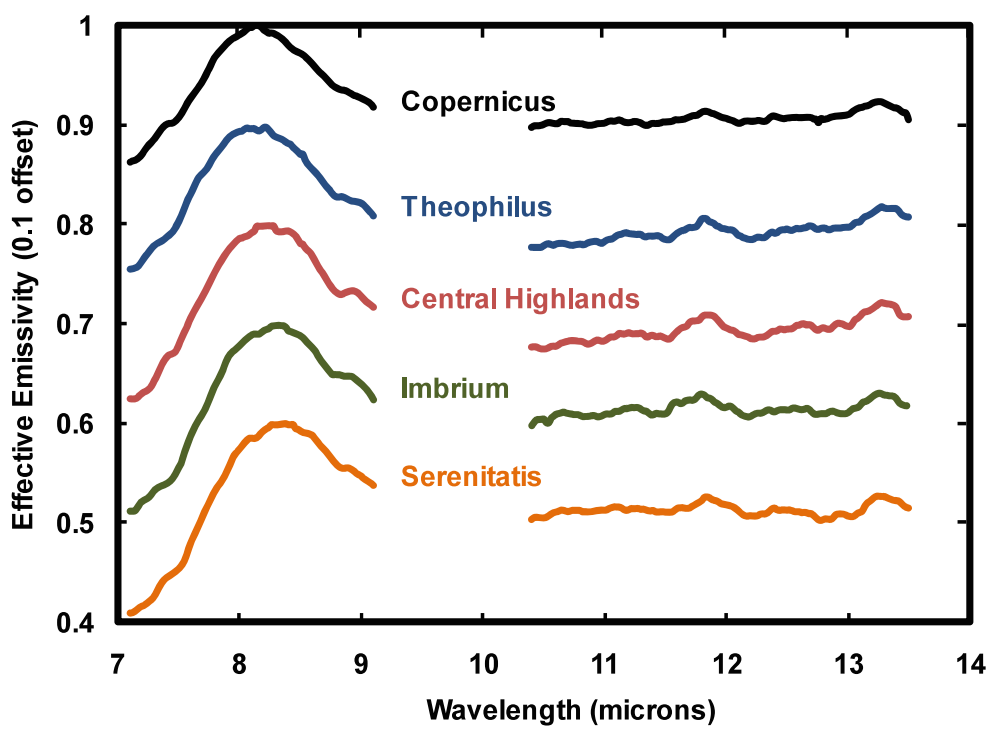

Fig. 4 Balloon-borne observations showing spatial variations in the lunar infrared emission spectrum from Murcray et al. (1970). The prominent emissivity maximum near 8.2 is the Christiansen feature (Salisbury et al. 1970)

an uneven distribution of regolith thermal properties and/or the presence of rocks (Murray and Wildey 1964). Balloon-borne telescopic measurements revealed the presence of a distinct peak in the Moon's thermal emission spectrum near $8 \mu \mathrm{m}$ which was interpreted to be the Christiansen feature, an emissivity maximum associated with $\mathrm{Si}-\mathrm{O}$ stretching vibrations (Murcray et al. 1970; Salisbury et al. 1970) (see Fig. 4).

Spacecraft missions to the Moon enabled more detailed infrared observations, in situ surface and subsurface temperature measurements, and determinations of the thermal and spectroscopic properties of lunar samples. Measurements and samples acquired at the Surveyor and Apollo landing sites confirmed the diurnal temperature behavior, and the general physical and spectral properties of lunar soils deduced from ground based measurements (Stimpson and Lucas 1970; Cremers and Birkebak 1971; Keihm and Langseth 1973; Logan et al. 1973; Salisbury et al. 1973).

The Apollo 17 Infrared Scanning Radiometer (ISR) experiment acquired thermal maps of approximately $25 \%$ of the lunar surface at a resolution of better than $10 \mathrm{~km}$ (Mendell and Low 1974). The instrument recorded brightness temperatures in a single uncooled thermopile bolometer channel with a spectral sensitivity of 1.2 to $70 \mu \mathrm{m}$ that ranged from 80 to $400 \mathrm{~K}$ with $\pm 2 \mathrm{~K}$ absolute precision. The data confirmed the presence of a large population of nighttime positive thermal anomalies or hot spots associated with the ejecta of small and large fresh impact craters (see Fig. 5). The accepted explanation for this phenomenon is that impacts excavate and expose blocky material with higher thermal inertia than the fine-grained lunar regolith. After exposure to the lunar environment over time, this blocky material is processed by smaller impacts and covered with fresh ejecta that gradually reduces its thermal inertia (Mendell 1976).

The Clementine Long Wavelength Infrared (LWIR) Camera mapped $0.4 \%$ of the Moon's surface from polar orbit with resolutions ranging from $200 \mathrm{~m} /$ pixel near the poles to 55 $\mathrm{m} /$ pixel at the equator (Lawson et al. 2000). The instrument employed a mechanically 


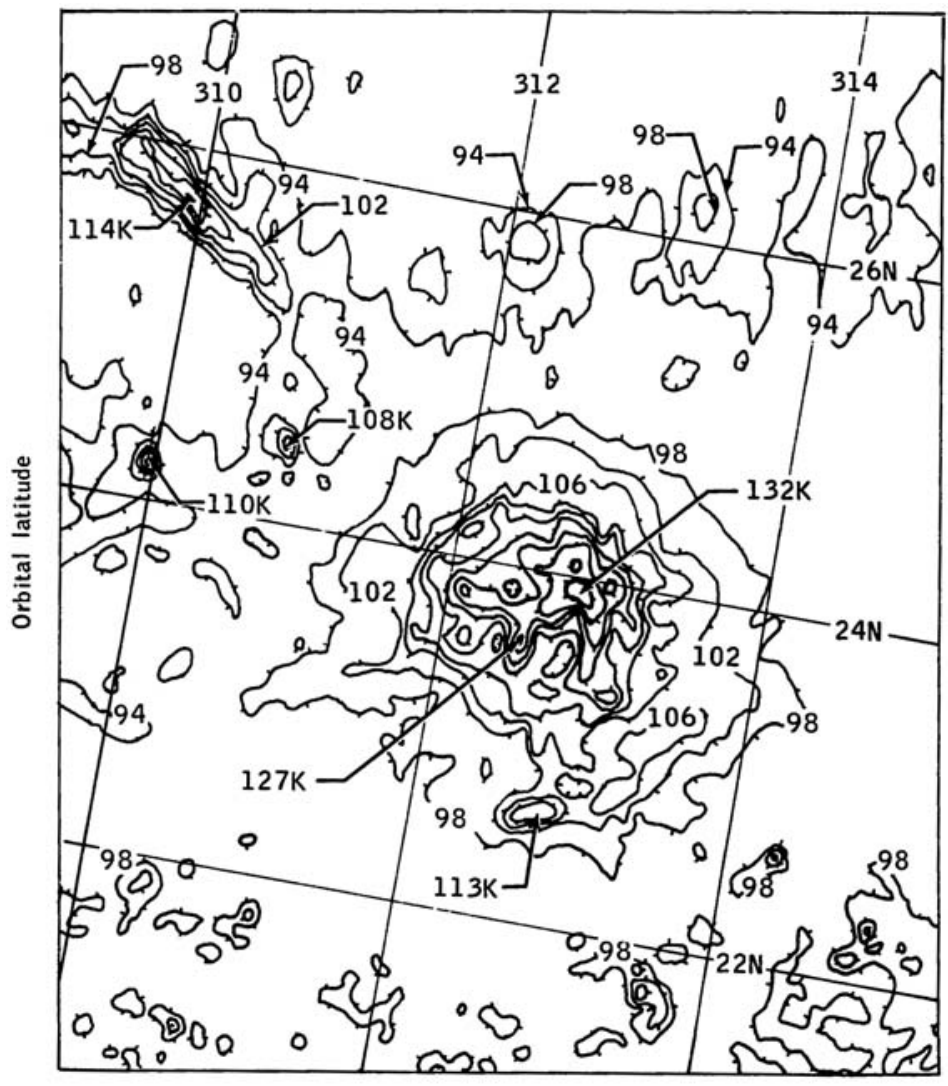

Orbital longitude

Fig. 5 Pre-dawn thermal map of the Aristarchus crater region by the Apollo 17 ISR (Mendell 1976). The contour interval is $4 \mathrm{~K}$. The $+32 \mathrm{~K}$ positive thermal anomaly associated with the interior of the crater has been attributed to the presence of exposed high thermal inertia blocks that were excavated during the relatively recent impact event that formed the crater

cooled $128 \times 128$ pixel $\mathrm{HgCdTe}$ detector array and had a single thermal infrared channel centered at $8.75 \mu \mathrm{m}$. Because the instrument's minimum detectable temperature was in the neighborhood of $200 \mathrm{~K}$, useful data were only acquired in directly illuminated regions. Since the lunar surface is in radiative equilibrium during the lunar day, and the rate of thermal emission is linearly proportional to the absorbed solar flux, most of the LWIR thermal images are indistinguishable from broad-band visible reflectance images of the Moon (Fig. 6).

Our current understanding of the lunar thermal environment is incomplete in a number of respects. While we have a good understanding of the bulk thermal properties of typical lunar regolith, and the large-scale diurnal thermal behavior at low and mid latitudes, we have much to learn regarding global thermal spatial variability, and its implications for lunar geological history. We also have good evidence for observable variations in the infrared spectral emissivity of the lunar surface, but the wavelength and spatial coverage of presently available observations has been insufficient to correlate with other compositionally-related datasets. We also have relatively little direct information regarding the thermal behavior of the Moon 


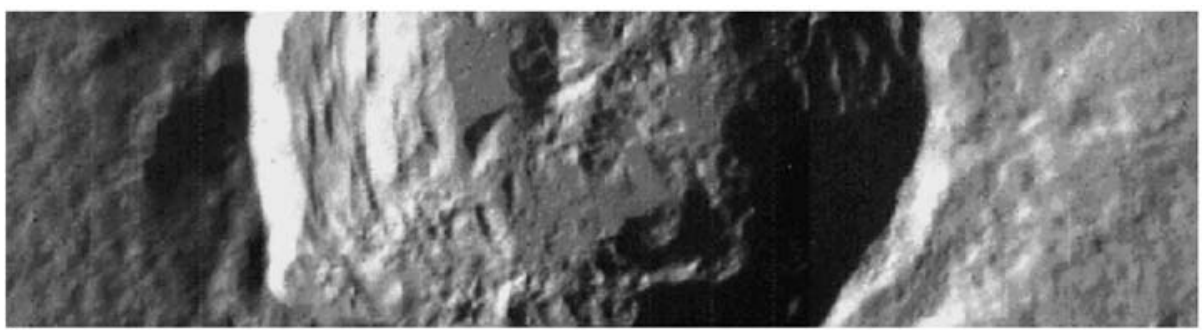

Fig. 6 Clementine LWIR daytime thermal image of Anaxagoras Crater $\left(75^{\circ} \mathrm{N}, 10^{\circ} \mathrm{W}\right.$; diameter $\left.52 \mathrm{~km}\right)$ at $\sim 200 \mathrm{~m}$ spatial resolution (Lawson et al. 1997), which is similar to the mapping resolution that will be achieved by Diviner

at smaller spatial scales - particularly in the polar regions where scattered solar and infrared radiation are the dominant heat sources. As described in the next section, the Diviner investigation will provide a significant new source of lunar thermal data that will provide a basis for an improved understanding of many aspects of the lunar thermal environment, as well as the implications for the Moon's geologic and volatile history.

\section{Investigation Description}

\subsection{History}

The Diviner Lunar Radiometer was conceived and proposed in response to NASA's LRO Measurement Investigations announcement of opportunity (AO) in 2004. The LRO AO solicited investigations that could provide temperature mapping of the Moon's polar shadowed regions, search for near surface water ice in the Moon's polar cold traps, and identify safe landing sites for future landed missions. Accomplishing these measurements would require a thermal mapping instrument with a low minimum detectable temperature, a small and well-defined field of view, and stable absolute calibration. At this same time, an instrument that possessed these same characteristics, the Mars Climate Sounder (MCS), was being integrated and tested for the Mars Reconnaissance Orbiter (MRO) mission at NASA's Jet Propulsion Laboratory (JPL). MCS was developed primarily as an atmospheric sounder, designed to scan the limb of Mars to obtain vertical profiles of temperature, dust and water vapor (McCleese et al. 2007). Like the earliest ground based infrared observers and the Apollo ISR, MCS employed un-cooled thermopile detectors that were coupled to a set of nine spectral filters that spanned a wavelength range from 0.3 to $50 \mu \mathrm{m}$. After some initial study, it became clear that a follow-on MCS instrument could meet and exceed the LRO measurement requirements with relatively minor changes to the MCS filter set, thermal design, software and electronics. Diviner, so named because of its potential ability to locate resources, was competitively selected as part of the original LRO payload in late 2004, designed and developed at the Jet Propulsion Laboratory, and delivered to the Goddard Space Flight Center for spacecraft integration in early 2008.

\subsection{Investigation Goals}

The goals of the Diviner investigation are driven by the exploration and science goals of the LRO mission. They can be summarized as follows: 
1. Characterize the Moon's surface thermal environments:
a) Daytime
b) Nighttime
c) Polar

2. Map properties of the lunar surface:
a) Bulk thermal properties
b) Rock abundance
c) Composition

3. Characterize polar cold traps:

a) Map cold trap locations

b) Determine their temperatures and thermophysical properties

c) Assess potential lunar volatile resources

\subsection{Investigation Approach}

Diviner is a nine-channel pushbroom radiometer designed to map emitted thermal radiation and reflected solar radiation from the surface of the Moon. Diviner will employ the following approaches to accomplishing the goals of the investigation:

\subsubsection{Characterizing the Moon's Thermal Environments}

Diviner will spend the majority of its time mapping the thermal emission from the lunar surface from low polar orbit. The goal will be to accumulate as consistent and complete a dataset as possible given the parameters of the LRO orbit and the duration of the mission, in order to characterize temperature variations as a function of latitude, longitude, time of day and season. Diviner will have the capability to make accurate radiometric measurements for the warmest and coldest surfaces on the planet, as well as simultaneous measurements of broadband solar reflectance. The Diviner dataset should be sufficiently complete to allow confident prediction of lunar surface temperatures in daytime, nighttime and polar thermal environments. Characterization of surface temperature variations at a given location over time will also place strong constraints on the thermal state of the lunar subsurface, which is of interest for a range of scientific problems and lunar exploration activities. Two immediate applications for this information would include constraining subsurface thermal conditions in suspected volatile cold traps at the lunar poles, and the definition of sites for future lunar bases and permanent habitats.

\subsubsection{Mapping Lunar Surface Properties}

Diviner radiometric temperature and solar reflectance measurements will be compared with the results of models to infer the bulk thermal properties of lunar surface materials and aspects of their composition. The combination of Diviner data and thermal models will enable mapping of the thermal inertia of the uppermost centimeters of the lunar soil, as well as the areal coverage of exposed rocks. While Diviner's spatial resolution will not be sufficient to resolve even the largest lunar ejecta blocks, the wavelength range of Diviner's infrared channels will enable determination of whether a given footprint includes surface materials emitting at differing temperatures, and estimation of the population of emitting temperatures within a given footprint. By analyzing patterns of spectral emission from the lunar surface over a complete diurnal cycle, it will be possible to map thermal inertia, rock abundance, surface roughness and infrared spectral emissivity at every location where sufficient 
Diviner coverage is obtained. In addition to new information regarding physical properties, Diviner's spectral emissivity measurements will provide new information regarding spatial variations in the composition of lunar surface materials that can be correlated with other lunar compositional datasets. The generation of Diviner's surface properties mapping products will benefit from the infusion of independently acquired topographic information at or below the spatial scale of the Diviner footprint. Such data, which can be acquired from orbital laser altimetry and stereo imagery investigations, will provide information regarding slopes and shadowing. The interpretation of Diviner's surface properties mapping products will be significantly enhanced through comparison with other mapped datasets relating to the composition and physical properties of the lunar surface.

\subsubsection{Investigating Polar Cold Traps}

The polar regions of the Moon are largely unexplored, and a number of significant questions remain regarding the possible existence of cold-trapped volatiles in the permanently shaded regions. The LRO payload includes multiple instruments that will contribute to a better understanding of these regions. Diviner's primary contribution will be to provide detailed thermal maps of permanently shadowed regions and adjacent areas. These maps can be used to determine the locations and surface temperatures of polar cold traps, and their diurnal and seasonal temperature variability that in turn, will place first-order constraints on the composition of volatile material that they may contain. Of particular significance is the potential correlation between the abundance of hydrogen in the uppermost lunar regolith and temperature. The Lunar Prospector (LP) Neutron Spectrometer data have strongly suggested the presence of enhanced hydrogen abundances in both the north and south polar regions. These data have been interpreted to be consistent with the presence of roughly $1.5 \%$ water-equivalent hydrogen in the uppermost $\sim 50 \mathrm{~cm}$ of the lunar regolith if concentrated in the floors of craters unresolved by the spectrometer's footprint (Feldman et al. 2001). The spatial resolution of the LP neutron data makes it difficult to uniquely establish the spatial distribution and concentration of the detected hydrogen. The LRO LEND instrument (Mitrofanov 2009) will acquire a higher-resolution dataset that should enable better localization of lunar polar neutron anomalies. Correlating compositional constraints provided by neutron datasets with thermal constraints provided by Diviner will make it possible to assess whether the neutron hydrogen signatures are localized within cold-trap locations, and correlate neutron hydrogen signature with cold-trap surface and subsurface temperatures. Enhanced hydrogen abundance in cold-trap locations would be strong evidence for the presence of cold-trapped water ice that would have important implications for our understanding of the volatile history of the Moon, and for future robotic and human exploration (Duke et al. 2006).

\section{Instrument Description}

The LRO Diviner radiometer (Fig. 7) is largely identical to the MRO MCS instrument described previously (McCleese et al. 2007). Key instrument specifications are listed in Table 1 . Two identical three-mirror off-axis telescopes are co-boresighted and mounted within an optical bench assembly. At the telescope focal planes are nine 21-element thermopile detector arrays, each with a separate spectral filter. The instrument will predominantly point in the nadir direction, operating as a multi-spectral pushbroom mapper. Two actuators, each allowing $270^{\circ}$ of rotation, provide pointing in azimuth and elevation. Diviner is mounted to 


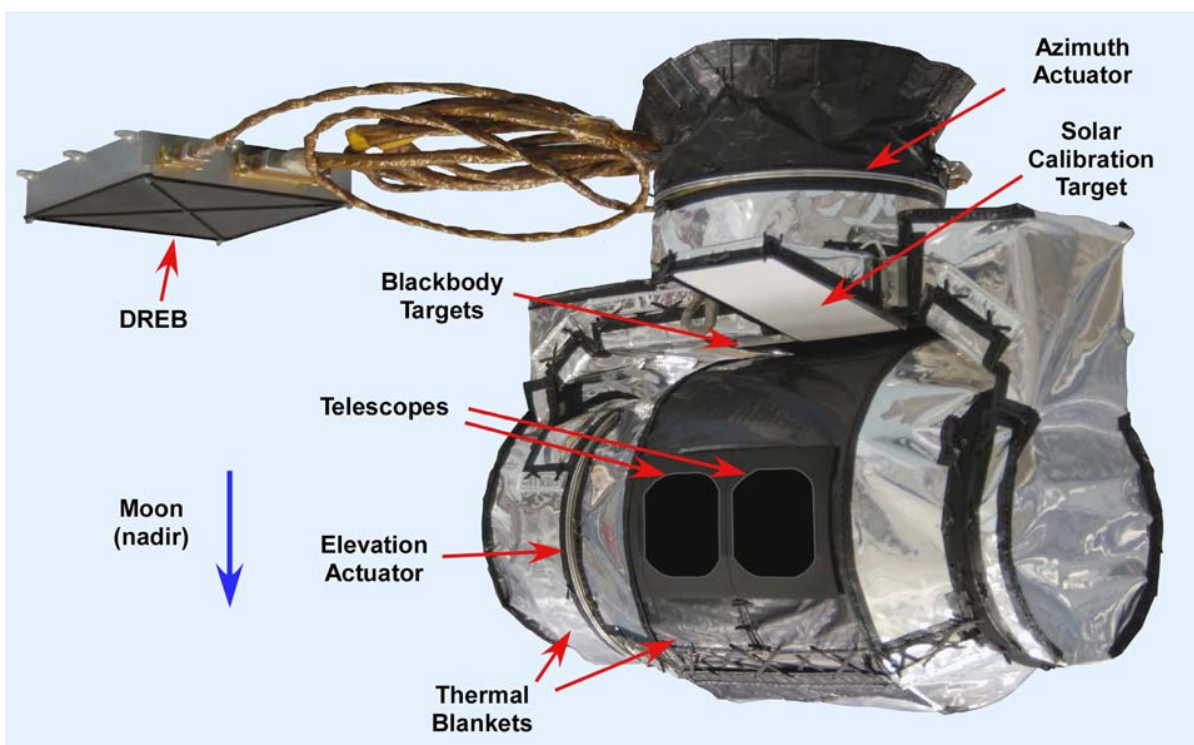

Fig. 7 Diviner instrument with major components labeled

the nadir panel of the LRO spacecraft, allowing it to view the Moon directly below. When the telescopes are pointed up into the instrument base they view a corrugated blackbody calibration target; when pointed horizontally they view space. The solar calibration target allows the solar channels to be calibrated using diffuse sunlight. The Diviner Remote Electronics Box (DREB) provides a Mil Spec 1553 interface to the spacecraft. The DREB also contains additional computing power compared to the MCS heritage design to allow the faster frame time required for pushbroom mapping.

Table 2 lists the nine spectral bands, their spectral passbands and measurement functions. In the text and figures they are often referred to by the channel names listed in the third column. Channels 1 and 2 measure reflected solar radiation from the lunar surface and utilize glass filters. These channels are referred to as the high- and reduced-sensitivity solar channels. Channels 3-5 use interference filters on zinc sulfide substrates provided by the University of Reading, UK. These channels, which have relatively narrow passbands near $8 \mu \mathrm{m}$, have sufficient signal-to-noise ratio to allow accurate spectral location of the emissivity maximum (Christiansen feature) for most known lunar materials at temperatures above $250 \mathrm{~K}$ (Greenhagen and Paige, 2006, 2009). Knowledge of the Christiansen feature spectral location provides compositional information. These channels are referred to as the $8 \mu \mathrm{m}$ channels. Channels $6-9$ divide the thermally emitted radiation roughly into four octaves, ranging approximately from 12.5 to $400 \mu \mathrm{m}$. Channel 6 uses an interference filter on a germanium substrate provided by the University of Reading. Channels 7-9 use stacks of hot-pressed copper mesh filters on polypropylene substrates provided by Cardiff University. Channels 6-9 are referred to as the thermal channels.

Channels 1-6 have a $\mathrm{KCl}$ window for long-wave blocking. Channel 7 has an interference filter and channels 8 and 9 have a quartz absorbing filter, both for short-wavelength blocking. All channels have a field-limiting aperture in front of the filter stack, running the length of the detector array and limiting the in-track field of view. The apertures for channels 2 and 6 are narrower than those over the other channels to avoid saturation at the highest signal 
Table 1 Diviner instrument specifications

\begin{tabular}{ll}
\hline Parameter & Property \\
\hline Instrument type & Infrared and solar radiometer \\
Spectral range & 0.35 to $400 \mu \mathrm{m}$ in nine spectral channels \\
Telescopes & Two identical three-mirror, off-axis, f/1.7 telescopes with $4 \mathrm{~cm}$ apertures \\
Detectors & Nine 21 -element linear arrays of uncooled thermopile detectors \\
& Pixel size $240 \mu \mathrm{m} \times 480 \mu \mathrm{m}$ \\
Fields of view & Detector Geometric IFOV: \\
& 6.7 mrad in-track \\
& 3.4 mrad cross track \\
& $320 \mathrm{~m}$ on ground in track for $50 \mathrm{~km}$ altitude \\
& $160 \mathrm{~m}$ on ground cross track for $50 \mathrm{~km}$ altitude \\
Swath Width $($ Center to center of extreme pixels): \\
67 mrad; 3.4 km on ground for $50 \mathrm{~km}$ altitude \\
Onstrument articulation
\end{tabular}

levels. These aperture widths are reduced to roughly $16 \%$ of the full width for channel 2 and $24 \%$ of the full width for channel 6 . These apertures reduce the in-track fields of view from the values listed in Table 1.

The channel layout in the two telescopes is shown in Fig. 8. The telescope A focal plane contains the six shorter wavelength channels. The longer wavelength mesh filters used in telescope B require more space for mounting, and hence only three filter channels fit on the telescope B focal plane. The channel colors used in Fig. 8 are the same as used in other figures in this paper.

Figure 9 illustrates where the Diviner spectral bands lie with respect to Planck blackbody emission from scenes at temperatures from 30 to $400 \mathrm{~K}$.

The internal calibration blackbody target (Fig. 10) consists of a grooved aluminum block coated with Martin Black. A blackened baffle assembly reduces stray light and divides the blackbody into two regions corresponding to the two Diviner telescopes. The baffle dimensions are slightly larger than the telescope aperture dimensions. The blackbody assembly is thermally isolated by ceramic spacers. Platinum resistance thermometers embedded in the aluminum block and a heater mounted on the backside of the target, are used for temperature control and measurement.

The solar calibration target (SCT) serves as Diviner's in-flight photometric calibration reference. The SCT is the last of five uniquely constructed targets originally created for Mars Observer Pressure Modulator Infra-Red Radiometer (PMIRR) (McCleese et al. 1986, 1992) and also used on MCS (McCleese et al. 2007). The SCT consists of a 6061 T6 aluminum base covered by an arc-sprayed coating of $99.999 \%$ pure aluminum that was grit blasted to remove specular facets (Ono 1999).

Diviner takes advantage of a great deal of heritage from the MCS instrument. Spare focal-plane assemblies from MCS were used. The vast majority of Diviner mechanical parts 
Table 2 Diviner spectral channel passbands and measurement functions

\begin{tabular}{|c|c|c|c|c|}
\hline $\begin{array}{l}\text { Channel } \\
\text { number }\end{array}$ & $\begin{array}{l}\text { Channel } \\
\text { type }\end{array}$ & Channel name & $\begin{array}{l}\text { Passband } \\
\mu \mathrm{m}\end{array}$ & $\begin{array}{l}\text { Measurement } \\
\text { function }\end{array}$ \\
\hline 1 & Solar & High Sensitivity Solar & $0.35-2.8$ & Reflected solar radiation, high sensitivity \\
\hline 2 & Solar & Reduced Sensitivity Solar & $0.35-2.8$ & $\begin{array}{l}\text { Reflected solar radiation, reduced } \\
\text { sensitivity }\end{array}$ \\
\hline 3 & $8 \mu \mathrm{m}$ & $7.8 \mu \mathrm{m}$ & $7.55-8.05$ & Christiansen feature \\
\hline 4 & $8 \mu \mathrm{m}$ & $8.25 \mu \mathrm{m}$ & $8.10-8.40$ & Christiansen feature \\
\hline 5 & $8 \mu \mathrm{m}$ & $8.55 \mu \mathrm{m}$ & $8.38-8.68$ & Christiansen feature \\
\hline 6 & Thermal & $13-23 \mu \mathrm{m}$ & $13-23$ & $\begin{array}{l}\text { Surface temperature (most sensitive } \\
\text { channel for }>178 \mathrm{~K} \text { ) }\end{array}$ \\
\hline 7 & Thermal & $25-41 \mu \mathrm{m}$ & $25-41$ & $\begin{array}{l}\text { Surface temperature (most sensitive } \\
\text { channel for } 69-178 \mathrm{~K} \text { ) }\end{array}$ \\
\hline 8 & Thermal & $50-100 \mu \mathrm{m}$ & $50-100$ & $\begin{array}{l}\text { Surface temperature (most sensitive } \\
\text { channel for } 43-69 \mathrm{~K} \text { ) }\end{array}$ \\
\hline 9 & Thermal & $100-400 \mu \mathrm{m}$ & $100-400$ & $\begin{array}{l}\text { Surface temperature (most sensitive } \\
\text { channel for }<43 \mathrm{~K} \text { ) }\end{array}$ \\
\hline
\end{tabular}

\section{Telescope A}
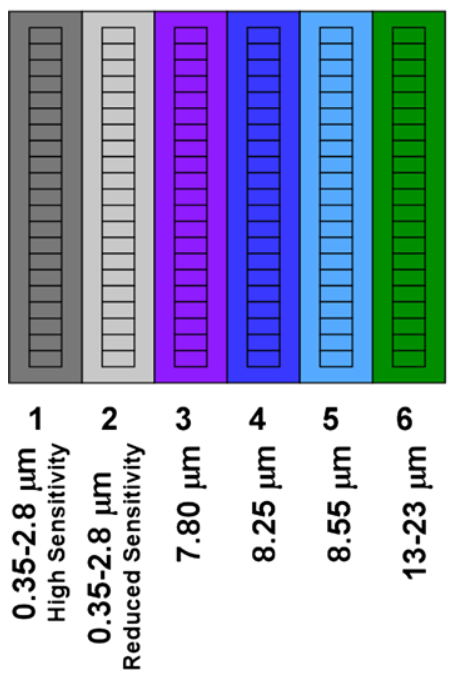

Telescope B

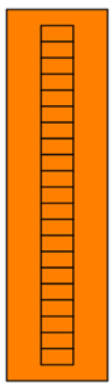

7

$25-41 \mu \mathrm{m}$

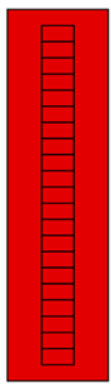

8

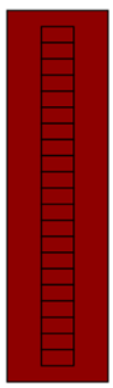

9

\section{$50-100 \mu \mathrm{m} \quad 100-400 \mu \mathrm{m}$}

Fig. 8 Diviner focal-plane layout. The two telescopes are co-boresighted but are separated in this figure for clarity

were build-to-print from MCS designs. A large fraction of the MCS electronics and software design was also reused for Diviner. However, several key changes, listed below, were made to enable Diviner to accomplish its lunar science goals. 


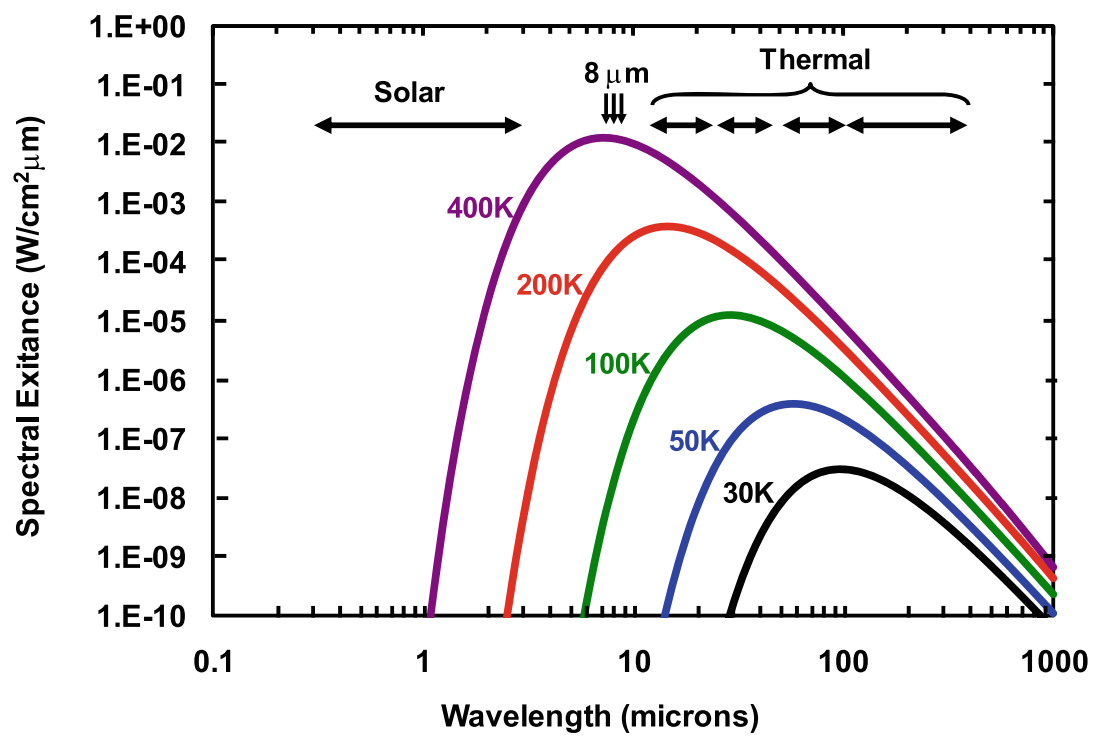

Fig. 9 The locations of the Diviner spectral channels and representative blackbody curves

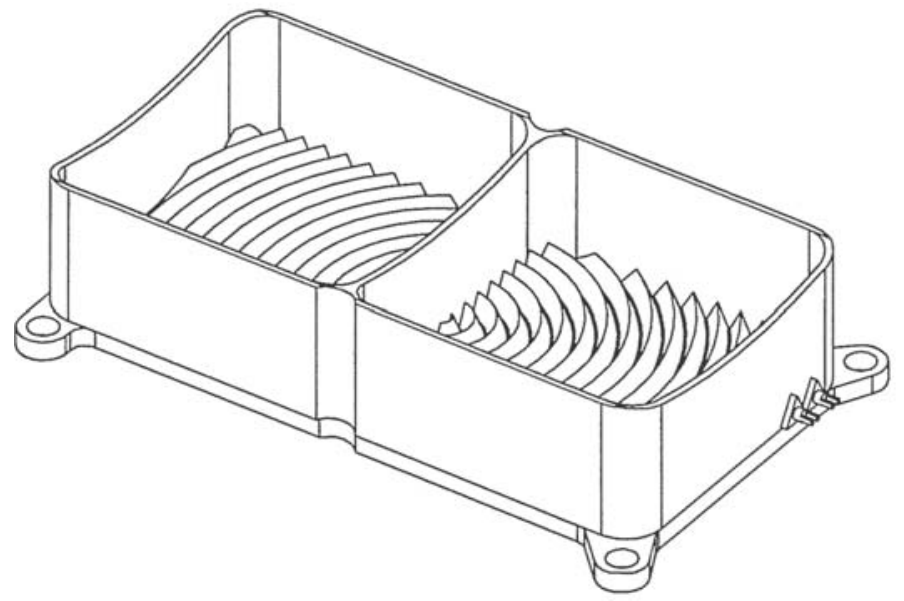

Fig. 10 Diviner internal calibration blackbody target

1. Diviner utilizes a different set of spectral passbands. The MCS bands were tuned to Mars atmospheric spectral features, while Diviner's bands are designed for measurements of lunar surface temperature, albedo, and the Christiansen feature.

2. Diviner requires a faster integration time to allow pushbroom mapping of the lunar surface. MCS was able to stare at the Mars limb for two second periods, while Diviner must integrate for only $128 \mathrm{~ms}$ to achieve the required spatial resolution and to minimize image smearing due to spacecraft motion. This change increases the data volume by roughly a factor of 16 and required numerous changes to the Field-Programmable Gate Array (FPGA) and software programs. 
3. The harsher thermal environment in lunar orbit compared to Mars dictated a modified thermal blanket and heater design. MCS has multilayer blanketing for insulation from the environment. The Diviner blanket is thinner, and some internal parts are painted black to allow more heat radiation to space. Additionally, an outer layer of silver Teflon was used over much of Diviner to reflect sunlight.

4. The DREB was added to provide a 1553 interface to the spacecraft (MCS used a lowvoltage differential signaling (LVDS) interface) and to provide additional computing power needed due to the sixteen-fold increase in data rate of Diviner compared to MCS.

An electrical block diagram is shown in Fig. 11. The LRO spacecraft provides three power circuits, one for instrument power, one for DREB electronics and supplemental heater power, and one for survival heater power. LRO also monitors five thermistors distributed through the instrument and DREB. A one-second time tick is supplied by the spacecraft through an RS422 interface. All other communication to and from the spacecraft occurs through the 1553 interface located in the DREB. The DREB and instrument communicate through an RS422 interface. The RS422 interface allows commands and time ticks to be sent from the DREB to the instrument, telemetry to be sent from the instrument to the DREB, and resets to be initiated by either the instrument or DREB. The instrument electronics provide science data collection, command and data handling, housekeeping (temperature and voltage monitoring), heater control, and actuator control and position sensing. The DREB provides additional science data processing as well as command and data handling.

\subsection{Observation Strategy}

Diviner employs a nearly continuous nadir viewing strategy. Minimizing gaps in nadir viewing enables accumulation of maximum spatial/temporal coverage for mapping. Although Diviner will spend much of its operational life in nadir staring, its MCS heritage provides a high degree of operational flexibility. The independent elevation and azimuth actuators are each capable of a $270^{\circ}$ range of motion divided into 2673 steps $\left(0.101^{\circ}\right.$ per step). Diviner can slew the elevation and azimuth actuators at rates of 25.1 and $23.9 \mathrm{deg} / \mathrm{sec}$, respectively. However, due to electronic crosstalk, Diviner cannot collect useful science data while slewing.

The pointing direction of Diviner is controlled by three types of tables through which the instrument software repeats continuously. The Orbit Schedule Table (OST) has twelve entries, each representing one orbit. The OST directs which Event Schedule Table (EST) will be used for each orbit. After the twelfth orbit, the OST repeats from the first entry. The EST controls activities over the course of a single orbit. The EST calls individual Scan Sequence Tables (SST) based on the time since the last equator crossing, which because of LRO's near polar orbital inclination, is effectively latitude. Each orbit, the LRO spacecraft generates and sends to Diviner a command containing the descending orbit equator-crossing time. The descending orbit will vary between day and night sides during the mission. An SST describes a frequently used scan pattern and includes multiple entries each of which addresses the following: (1) which actuator to move, (2) which step to move to, and (3) how long to stay at a given step. When an SST is completed, the first entry will be repeated continuously until the EST directs a new SST be used. The SSTs must be "sun safe" because Diviner's detectors and filters will be near-instantaneously destroyed if they cross the Sun.

Diviner routinely points at a number of locations, which are defined by specific actuator positions and ranges. These locations, highlighted in Fig. 12, include stowed, nadir pushbroom, internal blackbody calibration target, space view, and solar calibration target. The instrument stowed position, located at azimuth $0^{\circ}$ and elevation $0^{\circ}$, is the position where 


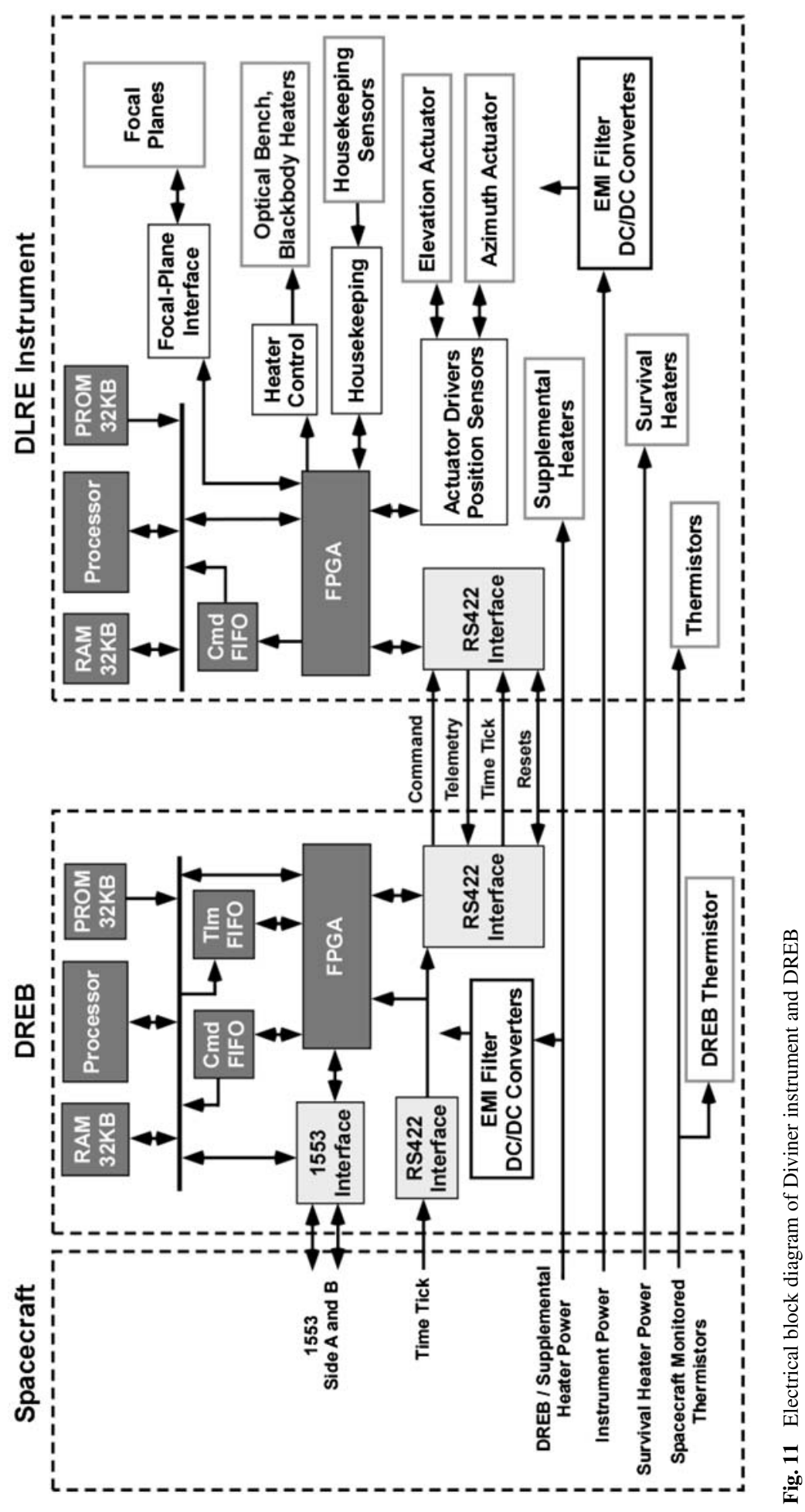




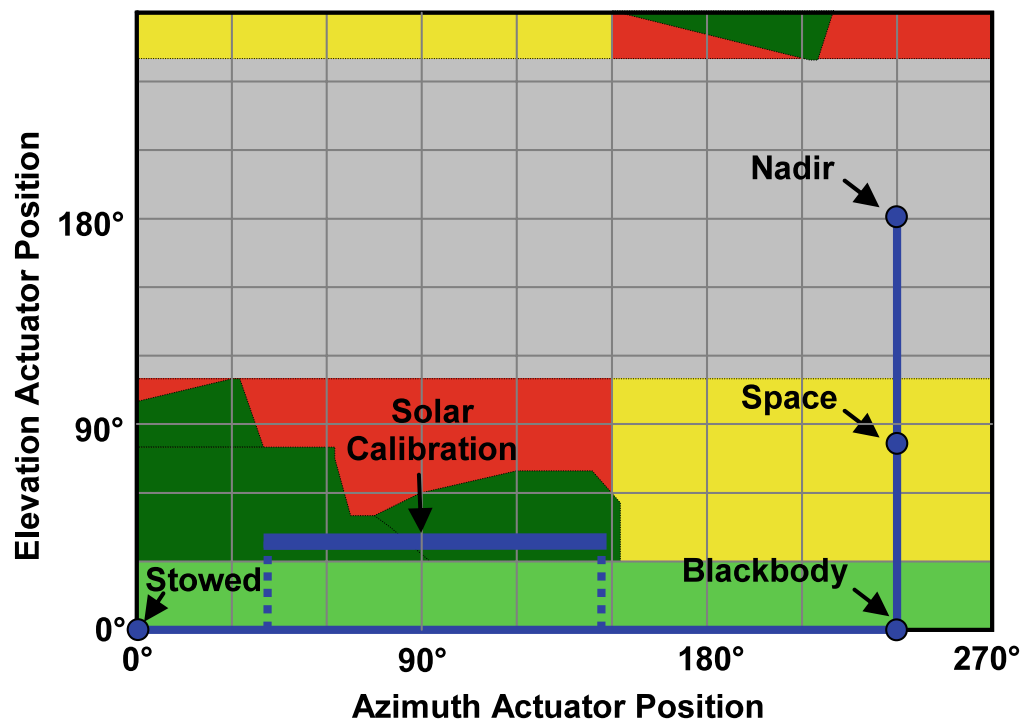

Fig. 12 The field of regard of the Diviner B telescope. The gray box represents the Moon for a $100 \mathrm{~km}$ circular orbit. The dark green areas are blocked by the spacecraft and instruments. The light green area is blocked by Diviner's yoke. Space regions are shown as both red and yellow: red is the region of space that could include the Sun, while yellow should normally never contain the Sun. Diviner will typically move along the blue line between common observation locations, nadir pointing, space views, blackbody calibration target, and stowed/safe position. The solar calibration target observations are made by the A telescope along the thick blue line between 40 and $150^{\circ}$ azimuth

the instrument starts after power on and resets and is the ultimate destination of all safing routines. The nadir pushbroom position is at azimuth $240^{\circ}$ and elevation $180^{\circ}$. The yokemounted internal blackbody calibration target can be observed at any azimuth with elevation $0^{\circ}$. Nominally, blackbody observations are made at azimuth $240^{\circ}$ in conjunction with nadir pushbroom mapping. Views of space, necessary for both in-flight radiometric and photometric calibrations are typically made at azimuth $240^{\circ}$ and elevation $80^{\circ}$. The solar calibration target can be observed at any azimuth with elevation $37^{\circ}$; however, the azimuth angle must be such that the target is illuminated.

\subsection{In-flight Calibrations}

Diviner performs routine two-point in-flight radiometric and photometric calibrations to calculate signal gain and offset values to correct for the effects of thermal and electronic drift. Both calibrations use space as the zero reference. The in-flight radiometric calibration uses the internal blackbody calibration target and the in-flight photometric calibration uses the solar calibration target mounted in front of telescope A (Channels 1-6) as the second reference. The solar calibration target was characterized pre-flight as described in a later section. The in-flight radiometric calibration sequence, which occurs approximately 10 times per orbit, is performed by pointing to space for 10 seconds, then pointing to the internal blackbody for 10 seconds, and then returning to space for 10 seconds. The total time required for the radiometric calibration is approximately 50 to 60 seconds depending on starting position. The in-flight photometric calibration sequence involves pointing to space for 10 seconds, then moving (in a sun-safe manner) to the solar calibration target position for ten seconds, 


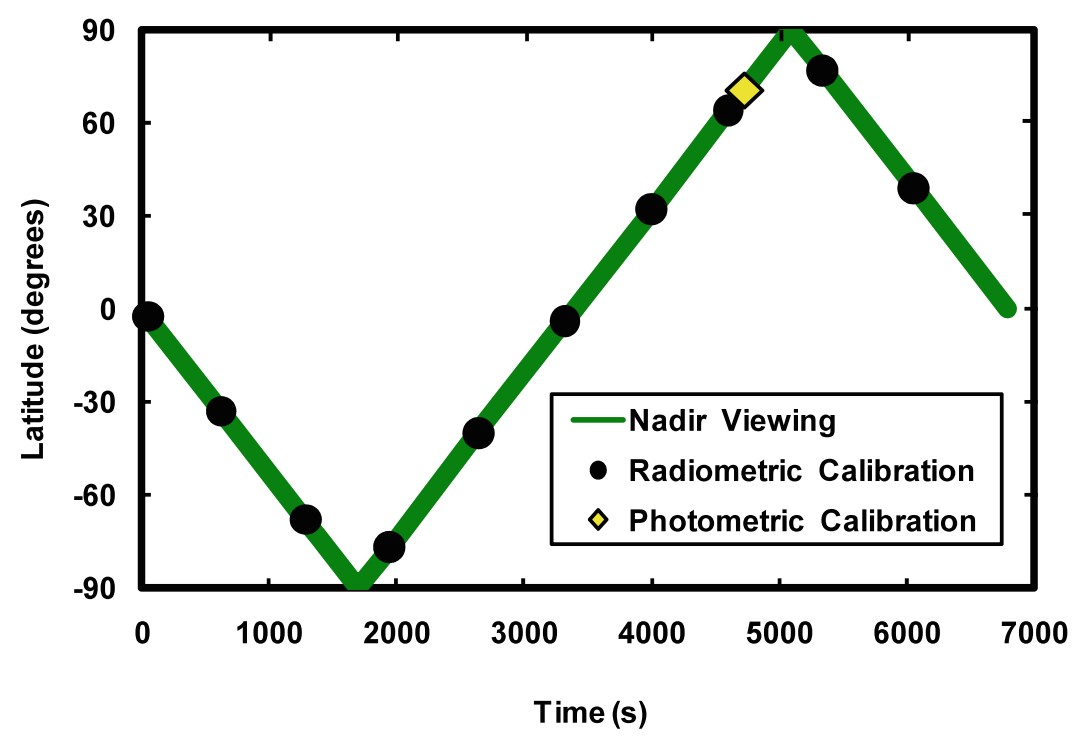

Fig. 13 Diviner observation and calibration activities during a typical LRO orbit. The timing of in-flight calibrations will be staggered to prevent latitudinal gores

then pointing to space for another 10 seconds. The total time required for the photometric calibration is approximately 75 to 85 seconds. The photometric calibration sequence is typically followed or preceded by a radiometric calibration and a duplicate space view is not performed. Values of gain and offset obtained through these calibrations are interpolated using a simple polynomial fit to obtain gain and offset values for each data sample.

There are several operational considerations regarding the timing and execution of the inflight calibrations. First, the in-flight radiometric and photometric calibration sequences are deviations from the nominal nadir staring and can cause latitudinal gaps in the spatial and temporal coverage (see Fig. 13, for example). Therefore, multiple ESTs will be employed to stagger the timing of the calibration sequences such that consecutive orbits do not have these gaps at the same latitudes. Weekly EST updates will incrementally change the latitudinal positions of the staggered calibrations. Second, the in-flight photometric calibration is ideally performed with the Sun low over the horizon to minimize additional radiation from sunlight reflected off the lunar surface and onto the solar calibration target. The latitude at which with these sun angles occur will vary throughout the mission (e.g. midnight/noon vs. terminator orbit). Finally, the azimuth position of the in-flight photometric calibration will be chosen to minimize sunlight reflected off Diviner itself and onto the solar calibration target. Bi-weekly updates will change the azimuth pointing of the photometric calibration.

\subsection{Spacecraft and Payload Considerations}

Diviner is programmed to automatically respond to a number of scenarios including large spacecraft rolls, Lunar Reconnaissance Camera (LROC) high resolution imaging, and spacecraft/instrument anomalies. Targeted narrow-angle LROC images may require the LRO spacecraft to roll up to $20^{\circ}$ off-nadir three times per day. During rolls, Diviner will execute its standard nadir viewing observation sequence to maintain its boresight relative to 
the other LRO instruments along the spacecraft $+Z$ axis. In-flight calibrations would continue even though some rolls will affect space and solar calibration target views. Testing may prove that Diviner's movements cause vibrations in the spacecraft sufficient to degrade LROC narrow angle images. For this reason, Diviner has the capability to "freeze", that is, to remain in its nadir position or to move there in a sun-safe manner. When frozen, Diviner will ignore scheduled calibration sequences while LROC narrow angle images are being acquired. Lastly, if there is an anomaly in the spacecraft or instrument, Diviner will execute a safing routine, abandoning its observation sequence and immediately moving to the stowed position in a sun-safe manner.

\subsection{LRO Orbit and Lunar Seasons}

The LRO orbit plane has an inclination of approximately $90^{\circ}$ relative to the lunar equator, and is nearly fixed in inertial space. As a consequence, the LRO sub-spacecraft longitude during the ascending or descending legs of the orbit will cycle between $0^{\circ}$ and $360^{\circ}$ once each month. Furthermore, the LRO sub-spacecraft local time during each orbit leg will cycle between 0 and 24 hours once each Earth year. Therefore, for nadir viewing geometry, Diviner will have an opportunity to observe a given point on the planet at a given local time twice each year, once on the ascending leg of the orbit and once on the descending leg.

The lunar spin axis is tilted at an angle of $\sim 1.54$ degrees relative to the ecliptic. As a consequence, the lunar arctic circles are located at latitudes of $\pm 88.46^{\circ}$ and insolation conditions at these highest lunar latitudes will depend on both local time and season. Because the Moon's orbital plane precesses with a period of $\sim 18.6$ years, the length of the Draconic Year (the period of the lunar seasonal cycle) is approximately 346.62 days, which is 18.13 days shorter than the 365.25 day Sidereal Year (the period of the orbit of the Earth and Moon around the Sun). Because the period of the Moon's rotation is a non-negligible fraction of its annual period, diurnal and seasonal insolation does not repeat exactly from one year to the next. For instance, the longitude that experiences maximum insolation (noon at summer solstice) will change each year. In order to observe maximum insolation conditions at the poles, the local time of the LRO Orbit will be roughly aligned with noon when solar declination is at its maximum. The degree to which this orbital alignment will be achieved depends on the exact launch date.

\subsection{Spatial and Temporal Coverage}

The percentage of the area of the Moon that Diviner will sample can be estimated using a statistical coverage model that assumes nadir pointing, average orbital altitudes and randomized ground track longitudes. At any given latitude, the fractional coverage, including random overlap, is $F=1-e^{-s n / c}$, where $F$ is the fractional coverage, $s$ is the swath width, $n$ is the number of latitude crossings by the orbit track (subject to various constraints), and $c$ is the circumference of the latitude circle. Figures 14 and 15 show calculated Diviner fractional coverage for nighttime observations (defined here to be between $7 \mathrm{pm}$ and $5 \mathrm{am}$ local time) and noontime observations (defined here to be 10 am to $2 \mathrm{pm}$ ). The calculations assume that Diviner will spend $85 \%$ of its time in nadir pushbroom mapping mode, with remaining fraction devoted to Diviner blackbody and solar calibration and off-nadir rolls by the LRO spacecraft. The LRO mapping orbit is assumed to be an ellipse with perilune altitude of $36 \mathrm{~km}$ over the south pole and an apolune altitude of $64 \mathrm{~km}$ over the north pole. The LRO extended mission orbit is assumed to be an ellipse with a perilune altitude of $30 \mathrm{~km}$ over the south pole and an apolune altitude of $206 \mathrm{~km}$ over the north pole. As Diviner's 


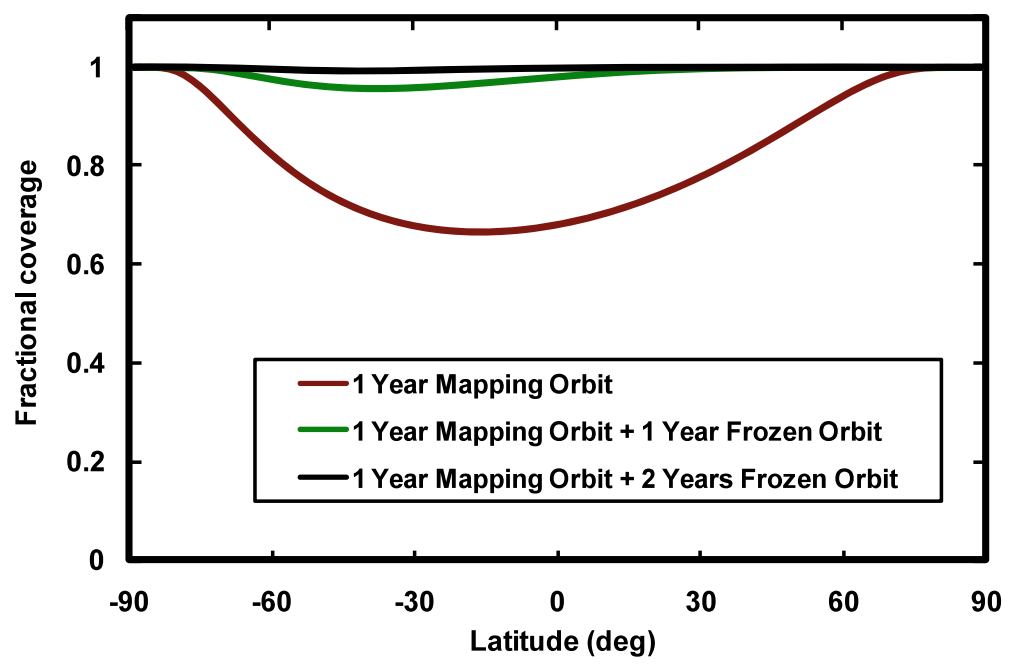

Fig. 14 Statistical model for the accumulation of Diviner nighttime coverage ( 7 pm to 5 am local time) during the LRO mapping and extended missions

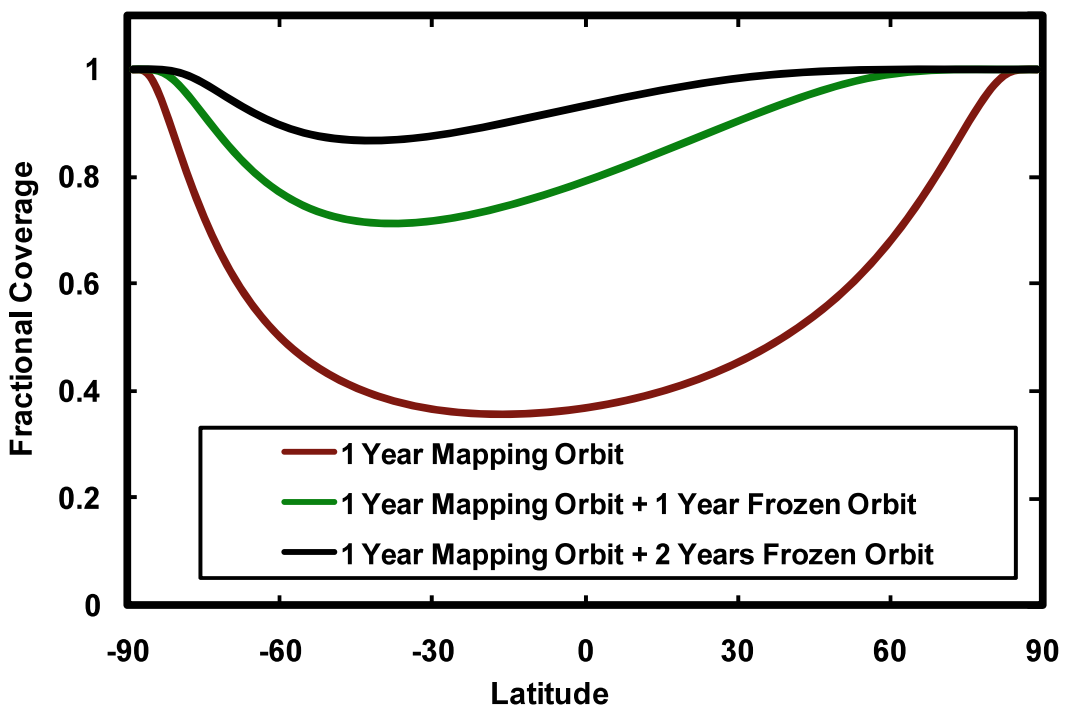

Fig. 15 Statistical model for the accumulation of Diviner noon-time coverage (10 am to 2 pm local time) during the LRO mapping and extended missions

swath width increases in proportion to the orbital altitude, higher altitude extended mission orbits will result in an increase in Diviner coverage in the north polar regions at the expense of spatial resolution.

During the one year LRO mapping mission, Diviner will acquire sufficient coverage to map the polar regions in detail, and provide extensive coverage of mid-latitude and equatorial regions. The nighttime coverage curves provide estimates of the percentage of the 
Moon's surface area over which Diviner will acquire observations suitable for estimating bulk thermal properties and rock abundances. The noon coverage curves provide estimates of the percentage of the Moon's surface area over which Diviner will acquire observations suitable for estimating surface emissivity and regions of permanent shadow. Averaged over a year, latitudes with good noontime coverage can also be interpreted as having good coverage at all local times. Diviner's extended mission coverage will enable the creation of more complete maps of the polar regions. Due to the higher eccentricity of LRO's extended mission orbit, Diviner's extended mission coverage of the north polar region will be at lower spatial resolution than during the primary mission, but it will still enable improved characterization of seasonal and diurnal variability. At equatorial and mid-latitudes, Diviner's enhanced extended mission coverage will fill most of the remaining coverage gores at the end of the primary mission, producing a nearly complete global dataset.

\section{Instrument Testing, Characterization and Calibration}

A full set of ground calibration and characterization testing was performed on the Diviner instrument, including measurements of radiometric response, spectral response, and the instantaneous field of view of every pixel. These measurements confirm that the flight instrument performs at levels that meet or exceed original design goals.

\subsection{Radiometric Response}

Diviner's laboratory radiometric calibration used two external blackbodies, which were scale copies of those used to calibrate the Improved Stratospheric and Mesospheric Sounder (ISAMS) instrument (Nightingale and Crawford 1991). The blackbody temperatures were monitored to an accuracy better than $0.1 \mathrm{~K}$. These blackbodies were positioned with their apertures within a few $\mathrm{cm}$ of the Diviner instrument such that movement of the elevation actuator could point the instrument into either external blackbody (Fig. 16). Each of the external blackbody apertures filled both of the instrument apertures. One of the external blackbodies (labeled Fixed Temperature Blackbody) was cooled to $90 \mathrm{~K}$ with liquid nitrogen. The second blackbody (labeled Variable Temperature Blackbody) was varied in temperature from 20 to $410 \mathrm{~K}$ using liquid helium, liquid nitrogen, and electrical heating. At each temperature setpoint of the variable blackbody, the instrument was scanned between the two

Fig. 16 Configuration used for laboratory radiometric calibration. The fixed temperature blackbody was maintained near $90 \mathrm{~K}$ using liquid nitrogen. The variable temperature blackbody varied in temperature from 20 to $415 \mathrm{~K}$
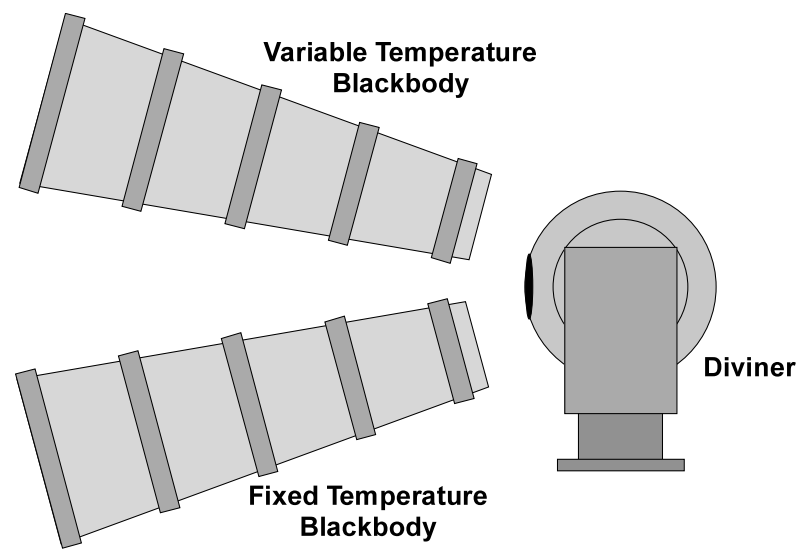


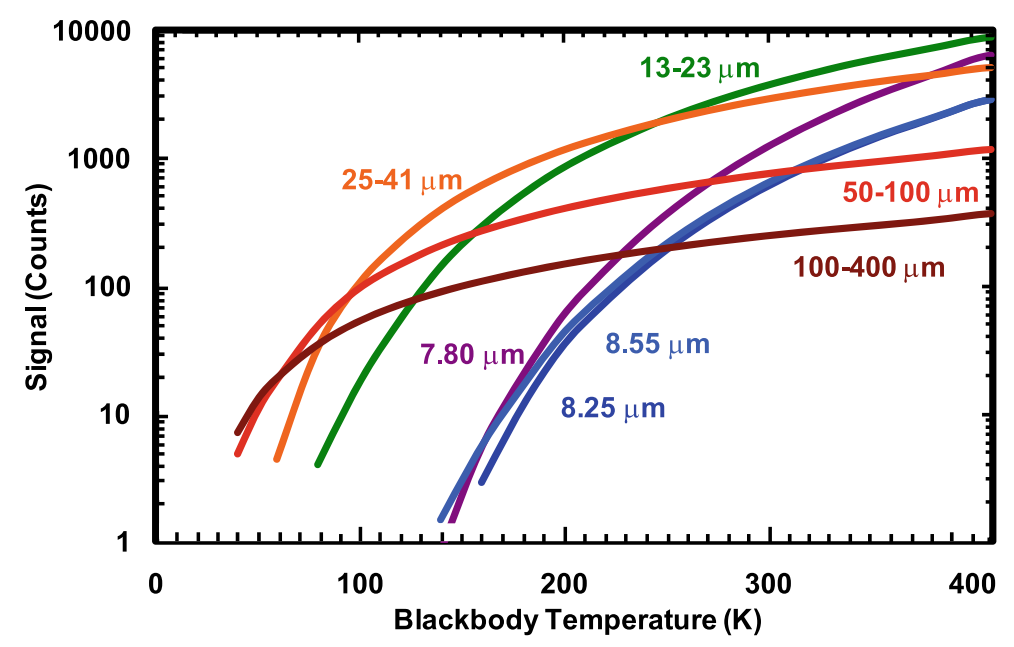

Fig. 17 Signal-to-noise ratio as a function of blackbody temperature for the thermal and $8 \mu \mathrm{m}$ channels

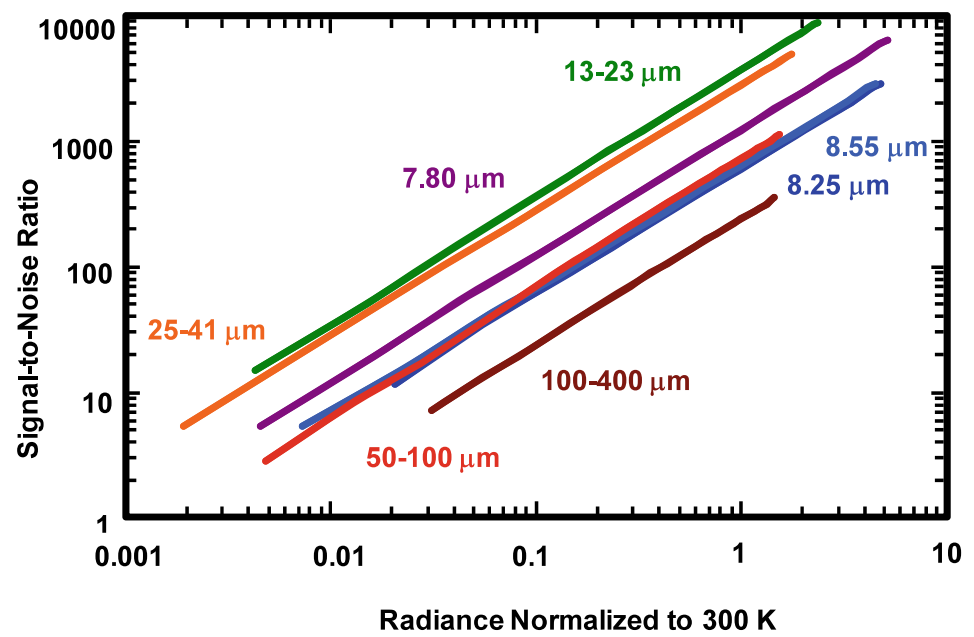

Fig. 18 Signal-to-noise ratio as a function of in-band radiance normalized to $300 \mathrm{~K}$ for the thermal and $8 \mu \mathrm{m}$ channels

external blackbodies and the internal blackbody multiple times. Using the fixed blackbody signal as a transfer standard, the signal at each variable blackbody temperature was referenced to the signal for a variable blackbody temperature of $20 \mathrm{~K}$, for which the signal in all channels is effectively zero.

The output signal-to-noise ratio from the instrument as a function of blackbody temperature for the thermal and $8 \mu \mathrm{m}$ channels is shown in Fig. 17. The same data set is plotted in Fig. 18 with radiance normalized to $300 \mathrm{~K}$ on the horizontal axis. Each trace in these figures represents the average of all 21 detectors in each spectral channel.

The radiometric calibration was performed over a range of instrument temperatures. The thermopile detectors have little temperature dependence. Changing the instrument tempera- 


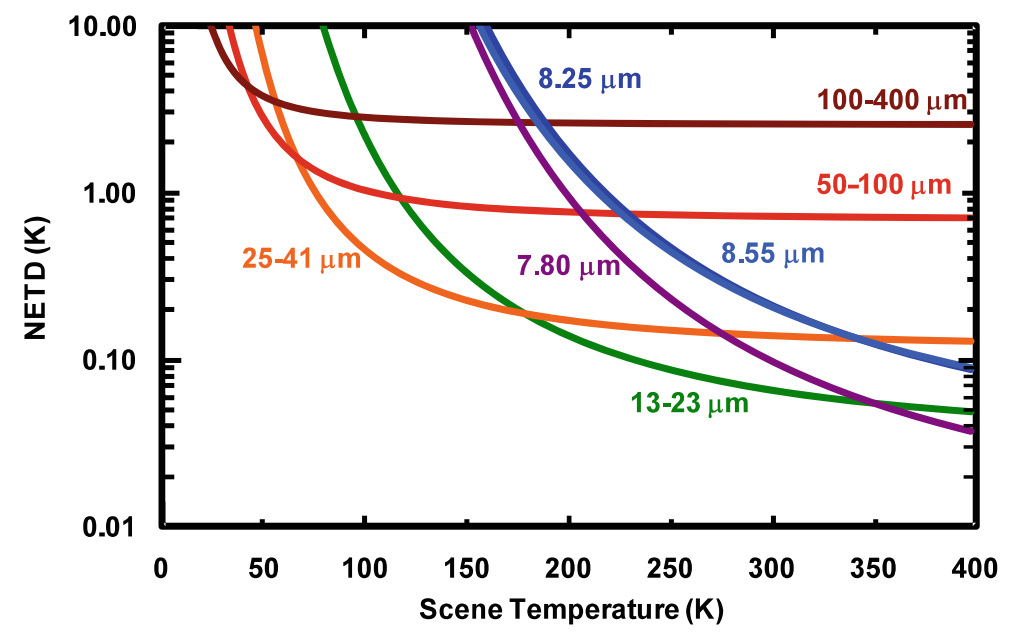

Fig. 19 Noise equivalent temperature for a single detector in each of the $8 \mu \mathrm{m}$ and thermal channels

ture changes the background radiation exchange between the detectors, the baffles, and the scene. In flight, the rate of this offset change is slow due to the instrument thermal design, and is removed by views of space every 10 minutes. Changes in detector and electronics gain are calibrated and removed by viewing the internal blackbody target in conjunction with the space views.

\subsection{Noise and Noise Equivalent Temperature Difference}

The detector noise level at the output of the signal chain is 2.5 counts with a standard deviation between pixels of 0.1 counts. The noise-equivalent temperature difference (NETD) for a single pixel in each of the thermal and $8 \mu \mathrm{m}$ channels is shown in Fig. 19.

\subsection{Radiometric Accuracy}

The uncertainty in a single radiometric measurement has components due to both offset and gain. Offset uncertainties in flight will be due to a combination of detector/electronics noise plus uncertainties in the interpolation of signals from space and blackbody looks at 10 minute intervals. A simulation of orbital conditions during thermal vacuum testing suggests that the uncertainty due to interpolation between space looks is roughly 2.5 counts. Assuming this uncertainty to be random, it can be combined with the uncertainty in detector/electronics noise ( 2.5 counts) by taking the square root of the sum of the squares, to result in a total offset uncertainty of 3.5 counts. This offset uncertainty is the dominant uncertainty source when looking at cold scenes.

There are numerous sources of gain uncertainty. During the ground radiometric calibration, uncertainty contributions include blackbody temperature measurements, non-unity blackbody emissivity, detector/electronics noise, and interpolation between points at different blackbody temperatures. In flight, additional uncertainties will occur from temperature measurement and non-unity emissivity in the internal calibration blackbody, detector/electronics noise and nonlinearity, and interpolation between gain measurements (space and blackbody looks) at 10 minute intervals. A complete and conservative analysis of these 
Table 3 Estimated in-flight gain uncertainties in the $8 \mu \mathrm{m}$ and thermal channels

\begin{tabular}{lll}
\hline $\begin{array}{l}\text { Channel } \\
\text { number }\end{array}$ & $\begin{array}{l}\text { Wavelength } \\
(\mu \mathrm{m})\end{array}$ & $\begin{array}{l}\text { Gain uncertainty } \\
(\%)\end{array}$ \\
\hline 3 & 7.80 & 1.7 \\
4 & 8.25 & 1.6 \\
5 & 8.55 & 1.6 \\
6 & $13-23$ & 0.9 \\
7 & $25-41$ & 0.6 \\
8 & $50-100$ & 0.6 \\
9 & $100-400$ & 1.1 \\
\hline
\end{tabular}

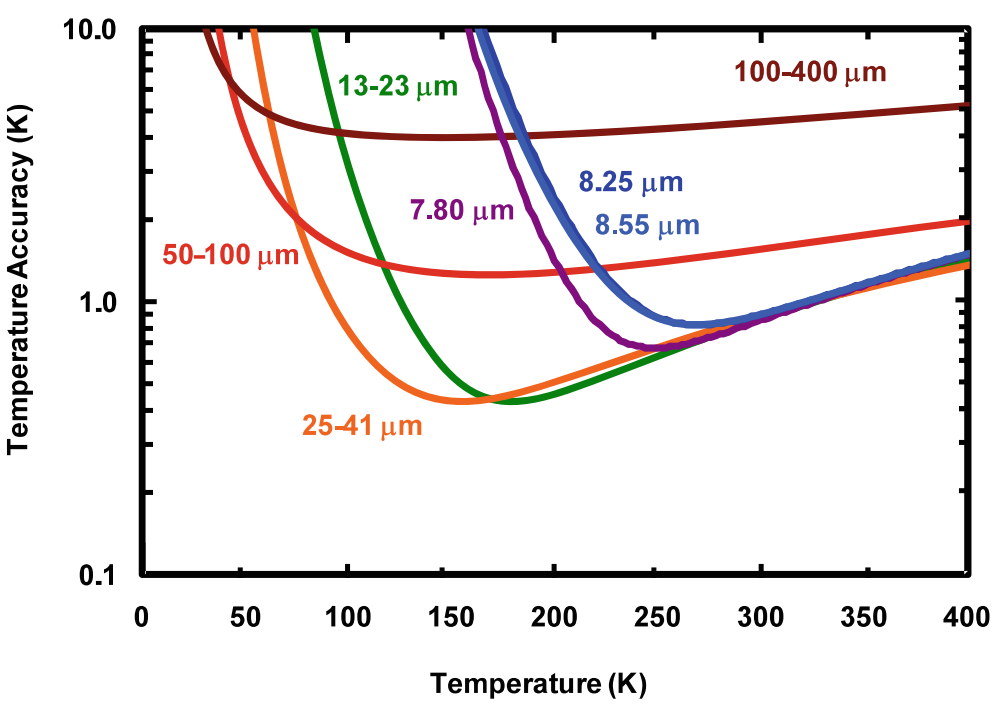

Fig. 20 Estimated temperature accuracy in $8 \mu \mathrm{m}$ and thermal channel measurements

various sources of uncertainty for the $8 \mu \mathrm{m}$ and thermal channels results in the estimated gain uncertainties listed in Table 3. Gain uncertainty dominates when looking at hot scenes.

The gain uncertainties in the $8 \mu \mathrm{m}$ channels will track each other because they are mostly due to ground and in-flight calibration uncertainty. Thus the uncertainty in the ratios of signals between these three channels will be much lower than the values shown in Table 3 . As determining the location of the Christiansen feature relies on these ratios rather than the absolute signals, the values used in this determination will be much more accurate than indicated in Table 3.

Figure 20 shows estimated temperature accuracy in the $8 \mu \mathrm{m}$ and thermal channels. The decrease in accuracy at low temperatures is due to offset uncertainty, while the decrease in accuracy at high temperatures is due to gain uncertainty. The results shown in Fig. 20 suggest that Diviner will acquire low noise, high accuracy radiometric measurements in multiple spectral channels over the full range of expected lunar surface temperatures. Higher signal-to-noise ratios may be obtained by averaging signals spatially or temporally, though accuracy is not always improved by this process due to systematic errors. 


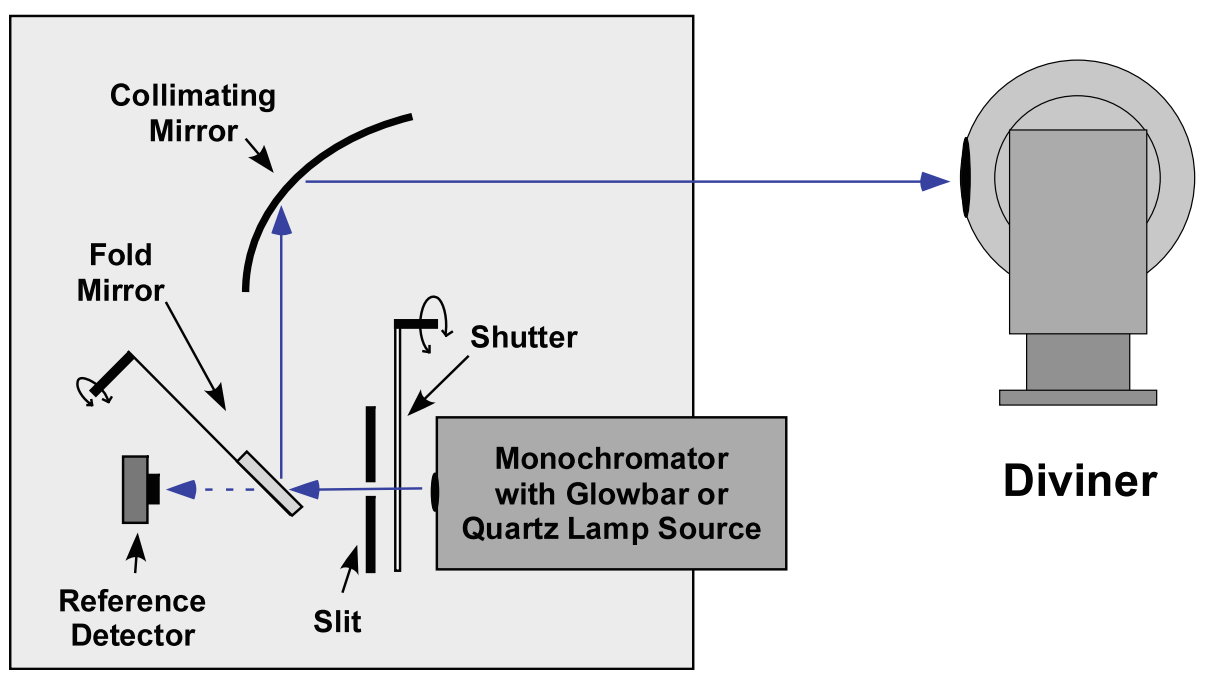

Fig. 21 Schematic diagram of spectral response measurement setup. The signal from the hot glowbar or quartz lamp goes through a slit, reflects off a fold mirror, and is collimated. A shutter behind the slit is used to block the source for background correction. The fold mirror can move to allow the signal to fall on a spectrally flat reference detector

\subsection{Spectral Response}

The spectral response in the solar channels, the $8 \mu \mathrm{m}$ channels, the 13-23 $\mu \mathrm{m}$ channel, and the $25-41 \mu \mathrm{m}$ channel was measured in the laboratory at the instrument level using a grating monochromator source (Fig. 21). The signal from the monochromator output slit was collimated, and then focused with the instrument optics onto a single detector in the instrument. A shutter behind the monochromator exit slit allowed subtraction of background signals. A fold mirror directed the monochromator signal either to the Diviner instrument or to a spectrally flat reference detector. A quartz lamp radiation source allowed measurements for $0.2-4.2 \mu \mathrm{m}$. A hot glowbar source was used for 2.6-56 $\mu \mathrm{m}$.

For measurements of the solar channels, eight scans were needed to cover the spectral range $0.2-4.2 \mu \mathrm{m}$ using the quartz lamp source. Between scans the gratings and/or ordersorting filters were changed. An additional scan from 3.6-6.8 $\mu \mathrm{m}$ was performed with the glowbar source. Within a scan, the relative spectral response shape is accurate. However, due to changing the gratings and order-sorting filters, adjacent scans are not expected to match exactly in the overlap region. To correct these mismatches, the data from each scan were manually shifted by as much as $50 \%$ in amplitude to form a complete seamless data set. The resulting errors of a few percent at the seams will introduce some error in the overall passband shape. Three detectors in the solar channels were measured over the full spectral range. These measurements were combined to form a single spectral response curve, shown in Fig. 22.

Figure 22 shows a significant spectral leak in the solar channels above $3 \mu \mathrm{m}$. Thus for warm scenes a fraction of the signal in the solar channels will be from thermal emission. Figure 23 shows an estimate of this thermal leak as a function of temperature. For this calculation, the lunar surface was assumed to be in radiative equilibrium with albedo 0.1. At $400 \mathrm{~K}$ roughly $3 \%$ of the signal in the solar channels will be due to thermal emission. To achieve accuracy in measurements of reflected solar radiation, a partial correction for this 

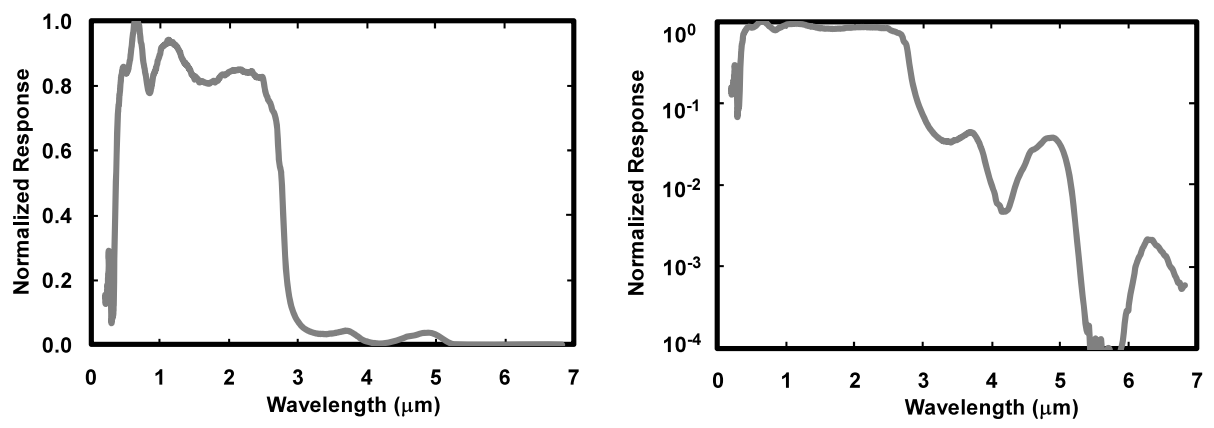

Fig. 22 Normalized spectral response of Diviner solar channels, linear vertical scale on left and logarithmic vertical scale on right

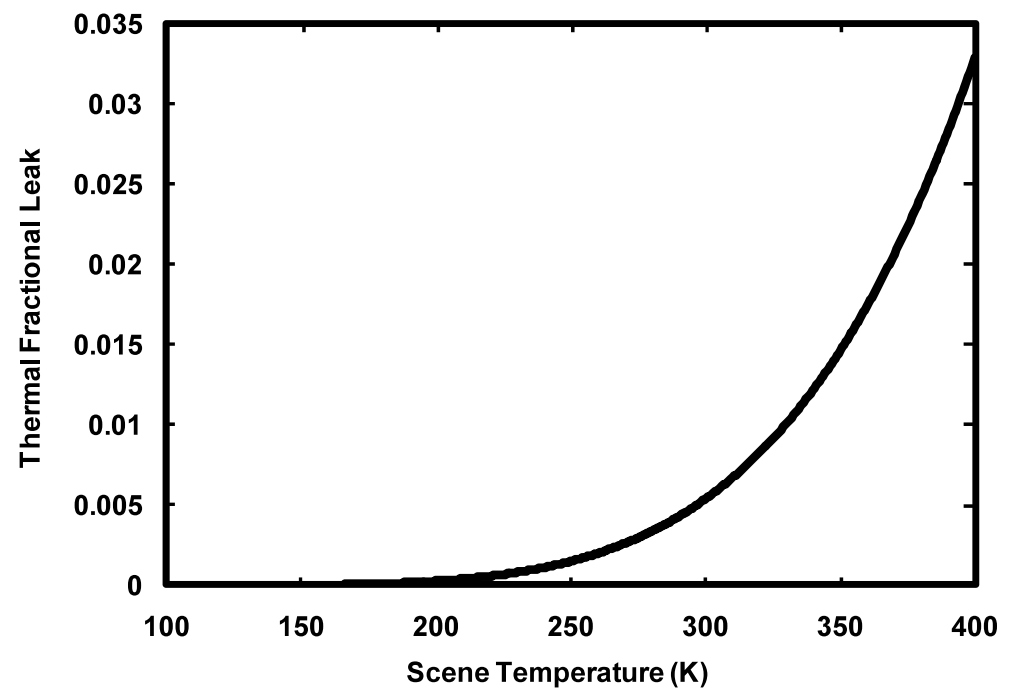

Fig. 23 Estimated contribution of thermal emission to signal in solar channels. Lunar surface is assumed to be an isotropic emitter and reflector in radiative equilibrium, with a solar spectrum averaged albedo 0.1

effect can be made using knowledge of the surface temperature from the thermal and $8 \mu \mathrm{m}$ channel signals.

The spectral response of the $8 \mu \mathrm{m}$ channels was measured from $6.5-9.8 \mu \mathrm{m}$ using a single monochromator grating and order-sorting filter, eliminating the need to stitch together data from multiple scans. Five equally-spaced detectors across each of the three arrays were measured. The passband variation across the detectors in a single channel was $25 \mathrm{~nm}$ or smaller. Because accurate spectral knowledge in these bands is critical for the determination of Christiansen feature location, a calibration check was performed on the monochromator in this wavelength region. A thin sheet of polypropylene was used as a transfer standard. The polypropylene was first measured with a Fourier transform spectrometer using an internal helium-neon laser reference. Then the output of the monochromator was measured using its reference detector, both with and without the polypropylene sheet in the beam path. The spectral features in the ratio of these two monochromator data sets matched the spectrometer 

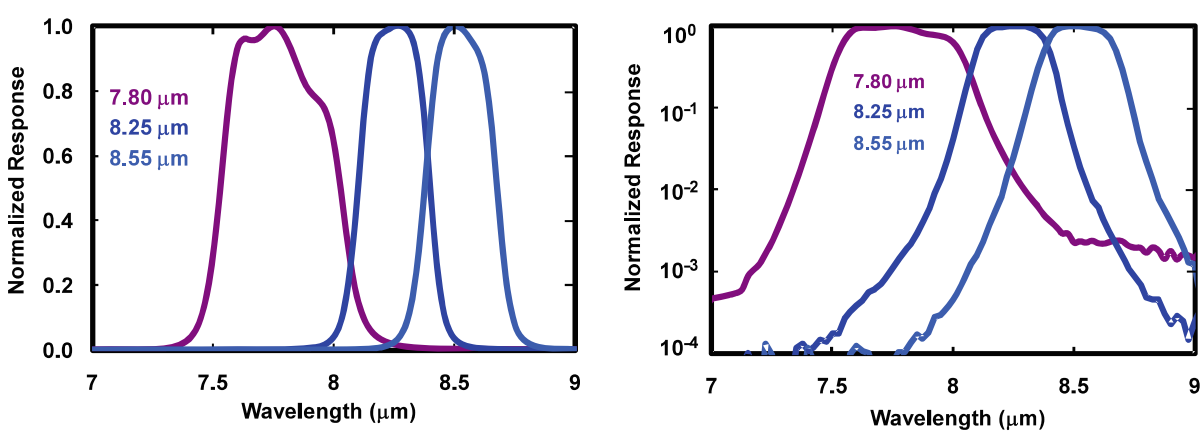

Fig. 24 Normalized spectral response of Diviner $8 \mu \mathrm{m}$ channels, linear vertical scale on left and logarithmic vertical scale on right
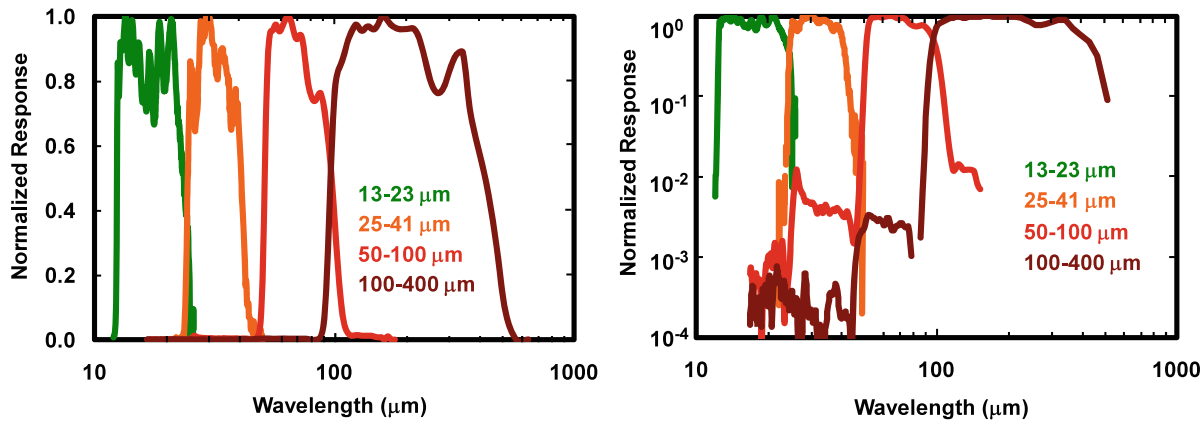

Fig. 25 Normalized spectral response of Diviner thermal channels, linear vertical scale on left, logarithmic vertical scale on right

polypropylene data to within a few nanometers, demonstrating sufficient monochromator spectral accuracy for this purpose. Figure 24 shows the spectral response of the three $8 \mu \mathrm{m}$ channels.

Spectral response measurements for the thermal channels were challenging due to limitations in monochromator signal at long wavelength. For this reason, only the 13-23 and 25-41 $\mu \mathrm{m}$ channels were measured at the instrument level. For these channels, data from multiple spectral ranges and multiple detectors were stitched together as in the solar channel measurements. For the 50-100 $\mu \mathrm{m}$ and 100-400 $\mu \mathrm{m}$ channels, the spectral transmission of the assembled filter stacks was measured with an $f$-number beam similar to the instrument. The resulting spectral response curves are shown in Fig. 25. The detector response is uncertain beyond $200 \mu \mathrm{m}$ and may very well roll off in this region. Thus the actual spectral response of the 100-400 $\mu \mathrm{m}$ channel is likely to roll off at long wavelength more quickly than shown in Fig. 25. Because the Planck distribution also rolls off in this range, the uncertainty in instrument response at long wavelength does not significantly affect its response to scene temperature.

The shapes of Diviner's radiometric response curves are in good agreement with model calculations of Diviner's radiometric response based on its measured spectral response. This provides independent evidence that there are no significant unanticipated spectral leaks that might complicate the interpretation of Diviner's thermal emission measurements. 
Fig. 26 Polar plot showing the solar-spectrum averaged reflectance of the Diviner solar calibration target relative to a normally illuminated perfectly reflecting diffuse reflector as a function of solar incidence angle (shown from 0 to $90^{\circ}$ ), and the solar azimuth angle. The open circles show the angles sampled by the goniometer measurements. The gray shaded region approximates the incidence and azimuth angles that are blocked by the Diviner instrument. The solid black rectangle shows the fixed viewing geometry of Diviner's solar channel detectors relative to the solar calibration target in emission and azimuth angle space. The solar calibration target was measured using six different fixed viewing geometries during pre-flight calibration

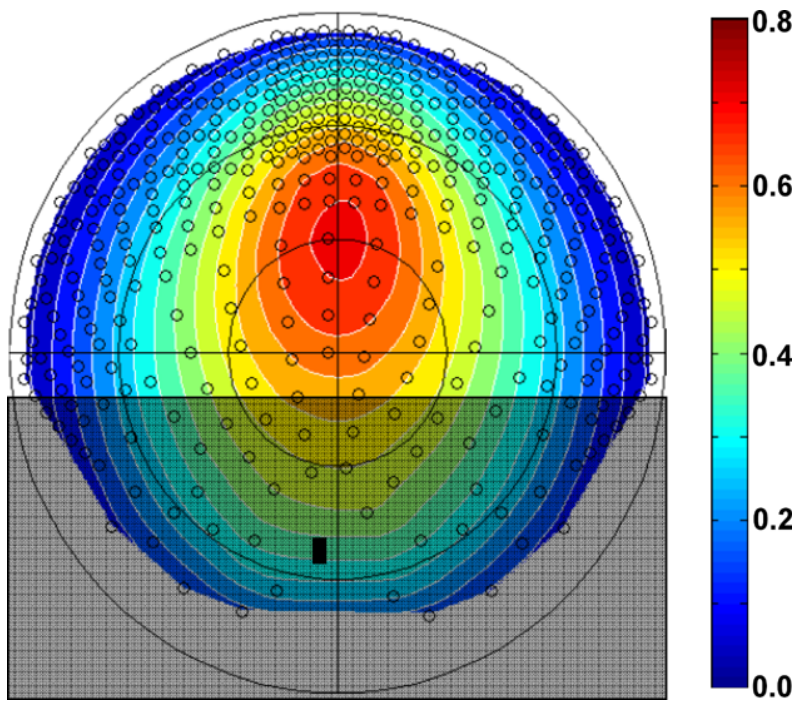

\subsection{Solar Target Calibration}

The hemispheric spectral reflectance of the Diviner solar calibration target was measured using a spectrophotometer coupled to an integrating sphere. The bi-directional reflectance of the solar calibration target was measured with a goniometer in 10 spectral channels in the wavelength region of $0.402-1.061 \mu \mathrm{m}$ at the same solar illumination angles and viewing geometry anticipated during flight. Diviner views the solar calibration target with constant viewing geometry so emission angle is effectively fixed, while solar incidence and azimuth angles can vary widely over the course of an orbit, and over the course of the mission. Figure 26 shows the solar spectrum-integrated bi-directional reflectance of the Diviner solar calibration target derived from the spectrophotometer and goniometer observations.

\subsection{Instantaneous Field of View}

The instantaneous field of view of each detector was measured in the laboratory using a target projector (Fig. 27). The target projector signal is generated by a hot target with a black painted surface. A slit wheel in front of the target allows one to change between narrow and wide vertical and horizontal slits without breaking vacuum. The image of the slit is collimated by a set of two mirrors. The target and slit are mounted on a sled, which has high-precision 3-axis actuators.

Two types of scans were performed with the Diviner instrument. For low-resolution scans the target projector sled stayed in a fixed position. These scans were performed by stepping the instrument actuators along the axis being measured. Each actuator step corresponds to $0.101^{\circ}$ or $1.76 \mathrm{mrad}$. High-resolution scans were performed over a narrower range of angles by fixing the Diviner actuator position at the center of the desired range and stepping the sled along the desired direction. Step sizes for high-resolution scans corresponded to $0.39 \mathrm{mrad}$ for in-track scans and 0.19 mrad for cross-track scans. 
Fig. 27 Target projector setup for instantaneous field-of-view measurements
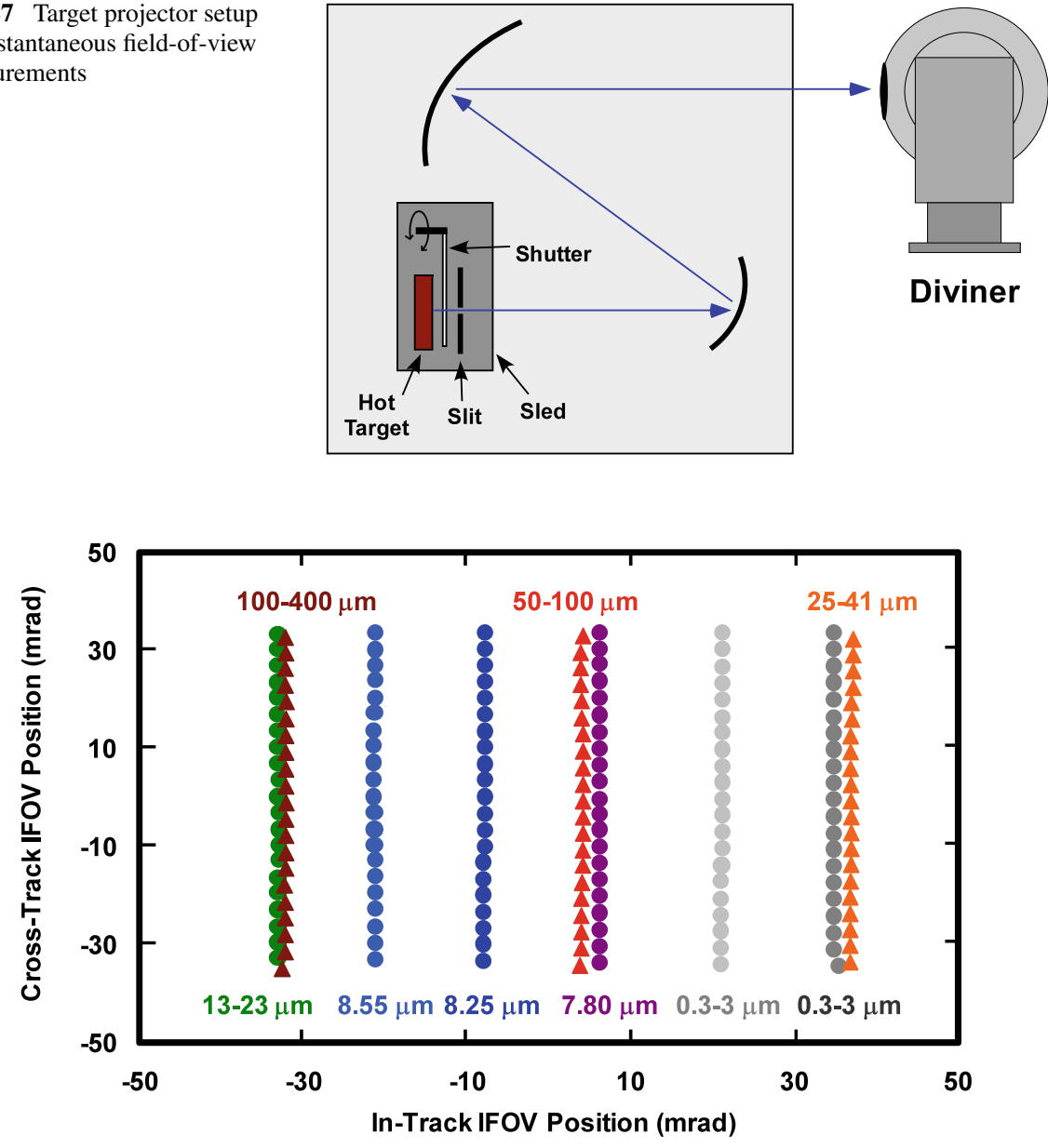

Fig. 28 Relative positions of field-of-view peaks for all Diviner detectors

Figure 28 shows a pixel position map in angular space, constructed from the peak positions determined from the low-resolution scans.

Figures 29 through 34 show the results of the high-resolution field-of-view scans. Each of these curves represents an average of all 21 detectors in a channel. For interpretation of lunar data, however, the unique instantaneous field of view for each detector will be used except in the 100-400 $\mu \mathrm{m}$ channel, where the signal-to-noise ratio in the field-of-view characterization is insufficient. For each group of channels (solar, $8 \mu \mathrm{m}$, and thermal), the fields of view are plotted both cross-track and in-track with both linear and logarithmic vertical scales.

In nearly all of the cross-track scans (see Figs. 29, 31 and 33), shoulders can be seen with a magnitude roughly $5 \%$ of the central peak. These shoulders are due to thermal crosstalk between adjacent detectors. Multiple shoulders of decreasing magnitude are present in some of the cross-track plots. The in-track fields of view of the reduced-sensitivity solar channel (Fig. 30) and the 13-23 $\mu \mathrm{m}$ channel (Fig. 34) are narrowed due to the reduced apertures over these spectral filters. 

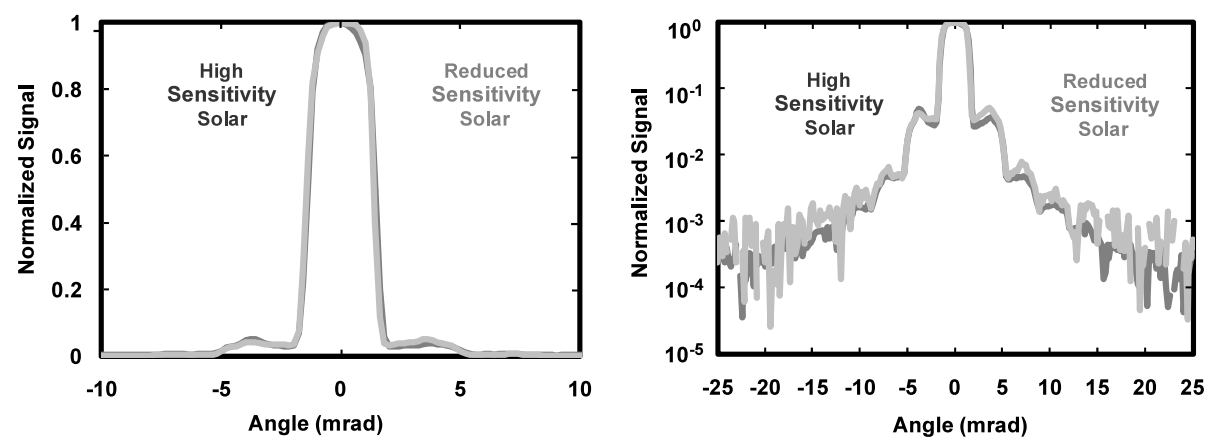

Fig. 29 Cross-track instantaneous field of view of Diviner solar channels, plotted with both linear and logarithmic scales
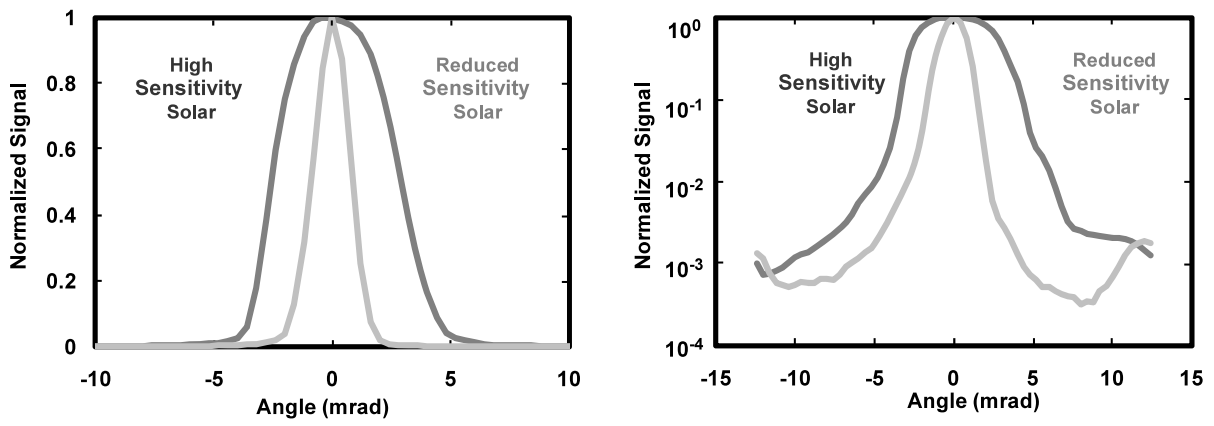

Fig. 30 In-track instantaneous field of view of Diviner solar channels, plotted with both linear and logarithmic scales
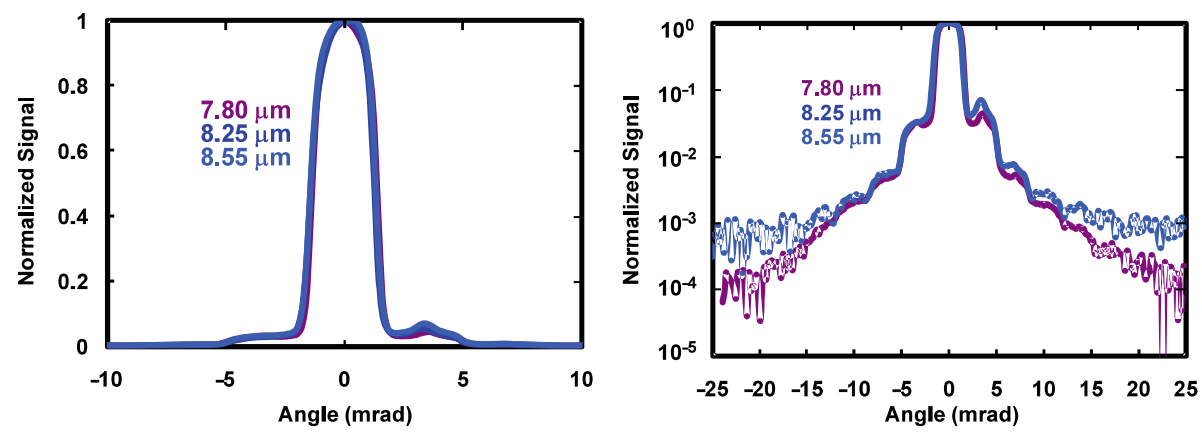

Fig. 31 Cross-track instantaneous field of view of Diviner $8 \mu \mathrm{m}$ channels, plotted with both linear and logarithmic scales

The three longest wavelength channels have the broadest fields of view (Figs. 33 and 34). Two effects in the mesh filters used in these channels contribute to this broadeningscattering and filter heating. Radiation transmitted through these mesh filters is scattered somewhat due to the filtering mechanism. In addition, the poor thermal conductivity of the polypropylene mesh support structure allows heating and cooling of the filters due to 

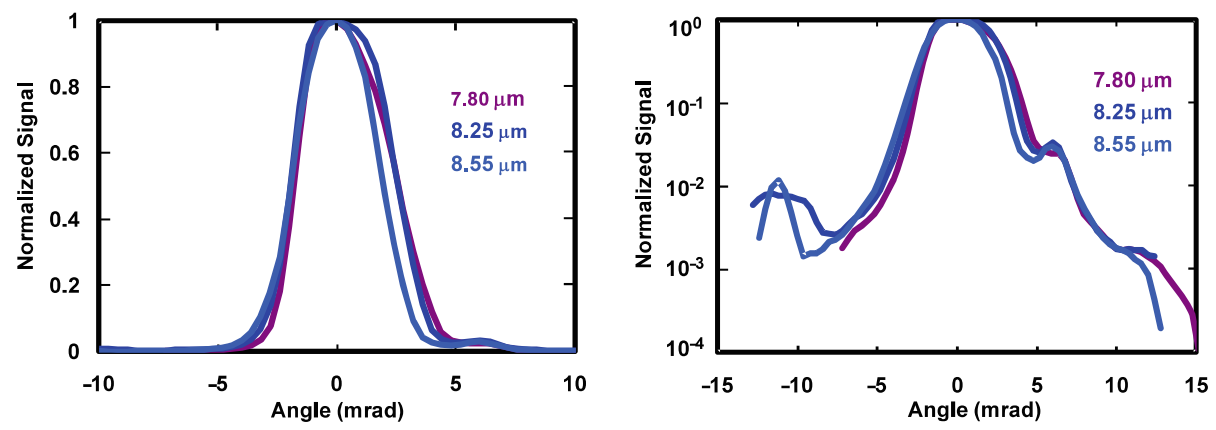

Fig. 32 In-track instantaneous field of view of Diviner $8 \mu \mathrm{m}$ channels, plotted with both linear and logarithmic scales
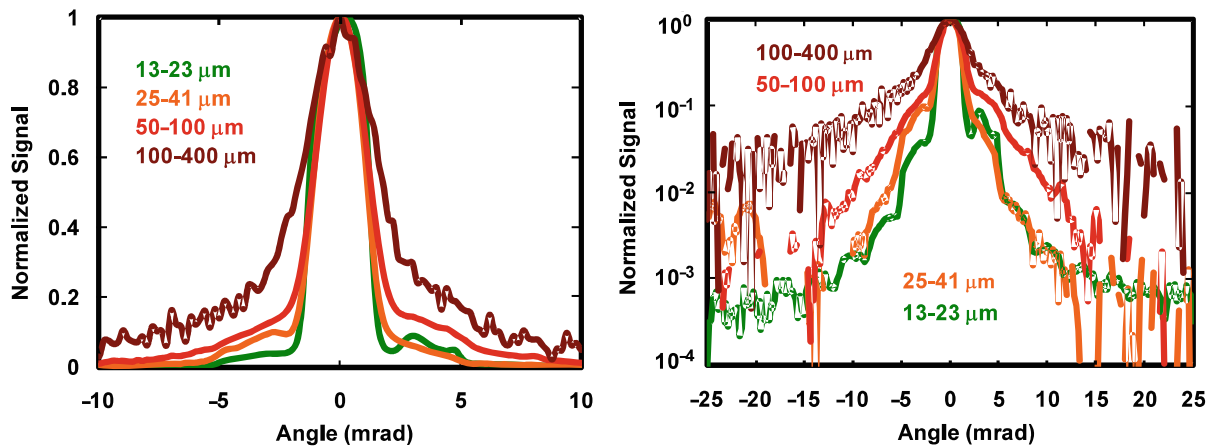

Fig. 33 Cross-track instantaneous field of view of Diviner thermal channels, plotted with both linear and logarithmic scales
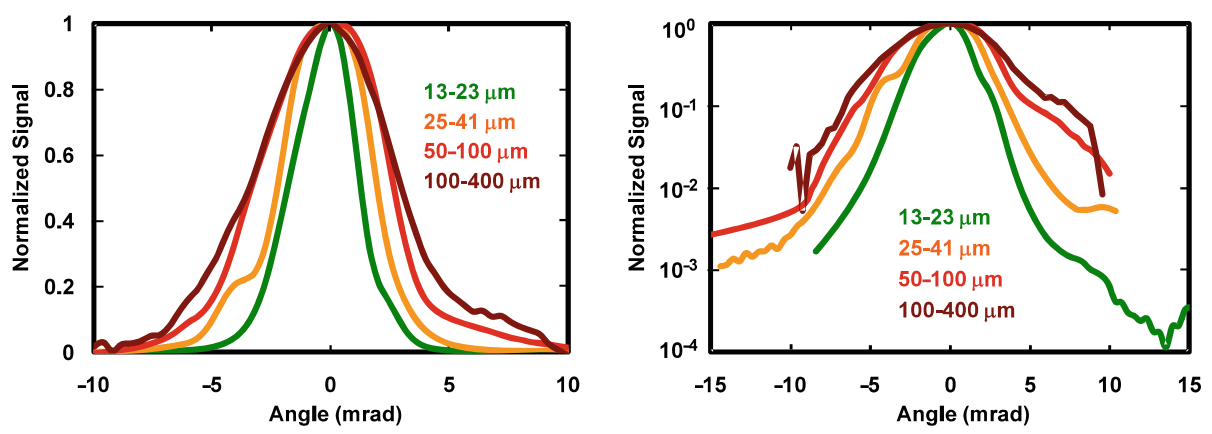

Fig. 34 In-track instantaneous field of view of Diviner thermal channels, plotted with both linear and logarithmic scales

changes in incident radiation. These temperature changes are conducted along the mesh structure, producing re-emission into neighboring pixels. Short-wave blocking elements in these filter stacks decrease these two effects significantly but do not completely eliminate 
them. Note that the increased broadening at the longest wavelengths is significantly more than would be expected due to diffraction.

Additional field-of-view information will be obtained in orbit by scanning across the lunar limb.

\subsection{Effective In-Track Field of View}

The effective field of view in the in-track direction is broadened by two effects: spacecraft movement during the $128 \mathrm{~ms}$ sample integration time, and the approximately $110 \mathrm{~ms}$ exponential detector thermal response time. The effective in-track field of view is the convolution of the instantaneous field of view (IFOV, shown in Figs. 30, 32 and 34) with a triangular function representing the integration time and with an exponential function representing the detector response time. The ground speed is approximately $1.66 \mathrm{~km} / \mathrm{s}$, varying only slightly with orbital altitude. Thus the smear caused by the integration time and detector response time corresponds to roughly $200 \mathrm{~m}$ on the ground. The footprint of the IFOV also varies linearly with orbital altitude. Therefore broadening of the in-track field of view is relatively larger at low altitude than it is at high altitude.

Note that in the cross track direction no field-of-view broadening occurs. The twodimensional effective field of view is oval, elongated in track due to the combination of a rectangular pixel, integration time, and detector response time.

Figure 35 shows the in-track instantaneous field of view for the $8.25 \mu \mathrm{m}$ channel as well as the two functions representing broadening due to integration time and detector response time for a $50 \mathrm{~km}$ altitude. All three functions are normalized to have a maximum value of one. The direction of the response time broadening depends on the spacecraft velocity direction. When the spacecraft performs a $180^{\circ}$ yaw maneuver every six months to keep the Sun on the same side, the velocity reverses with respect to the IFOV. The normalized

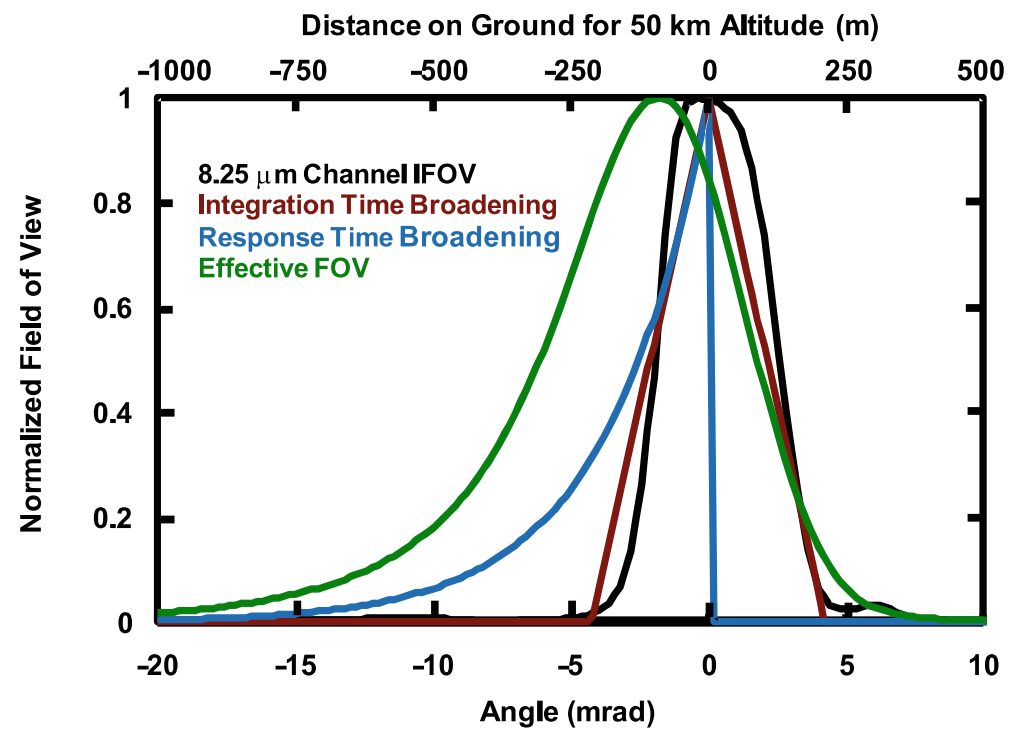

Fig. $358.25 \mu \mathrm{m}$ channel in-track IFOV plotted with broadening functions due to integration time and detector response time for a $50 \mathrm{~km}$ altitude. The green curve is the effective field of view resulting from the convolution of the other three functions 

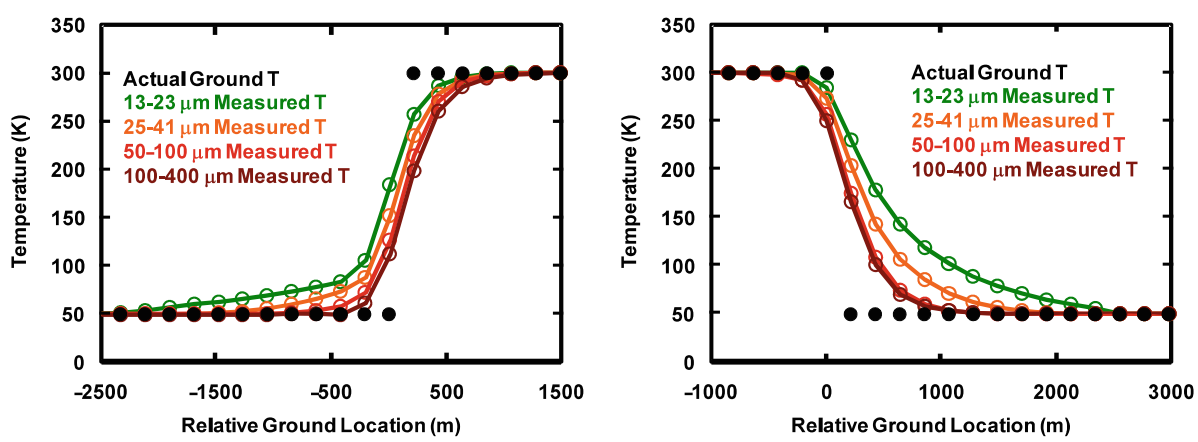

Fig. 36 Simulated Diviner thermal-channel response to a temperature step from 50 to $300 \mathrm{~K}$ (left) and from 300 to $50 \mathrm{~K}$ (right). Direction of travel is from left to right

convolution of these three functions (effective FOV) is also shown in Fig. 35. Note that the effective field of view for a given pixel is not fixed with time, but rather depends on the spacecraft altitude and velocity vector.

\subsection{Response to a Step Function in Scene Temperature}

With knowledge of the effective field of view of the pixels in each channel, one can calculate the instrument response to a step function in temperature. A large, sudden step in scene temperature could occur at a crater rim for example, in which one side is illuminated by the Sun and the other side is in shadow. While looking at one side of this step, part of each pixel's effective field of view sees the much different temperature on the other side of the step. The contamination caused by the nearby scene depends both on the magnitude of the field-of-view tails and on the way the response of that spectral channel depends on wavelength.

Figures 36 shows simulated Diviner data of in-track temperature transitions from 50 to $300 \mathrm{~K}$ (left) and from 300 to $50 \mathrm{~K}$ (right). The two results are not mirror images of each other because of the asymmetry in effective field of view due to detector response time (Fig. 35). The shortest wavelength channel (13-23 $\mu \mathrm{m})$ has the most leakage of the warm signal into the cold region. This channel is the most nonlinear as a function of temperature, with response strongly weighted towards the higher temperatures (see Fig. 17). The longest wavelength channel, in contrast, has nearly linear response as a function of temperature. This channel has roughly equal leakage in both directions.

The Diviner's spatial response has been sufficiently well characterized to enable confident localization of surface thermal features at spatial scales that approach the field of view of the instrument. Enhanced ground processing may make it possible to deconvolve the angular and temporal smear that is inherent in the raw observations to provide even more spatially resolved and more accurate temperature maps of regions of high scientific interest.

\section{Data Analysis and Interpretation}

\subsection{Standard Data Products}

The Diviner team will create a series of standard archived data products for NASA's Planetary Data System as shown in Table 4. Level 0 and Level 1 data products will be archived 
Table 4 Diviner archived data products

\begin{tabular}{ll}
\hline Archived data product & Description \\
\hline Pre-flight Calibration Data & $\begin{array}{l}\text { Pre-flight calibration data (Spectral response, blackbody response, solar target } \\
\text { reflectance, fields of view, alignment) }\end{array}$ \\
Experimenter's Notebook & Chronologic text description of instrument operation and performance \\
Level 0 & Time-sequenced raw science and housekeeping data \\
Level 1 & $\begin{array}{l}\text { Calibrated radiances and housekeeping data merged with project-supplied } \\
\text { geometry and timing data }\end{array}$ \\
Level 2 & $\begin{array}{l}\text { Gridded global brightness temperature (latitude, longitude, local time, season) } \\
\text { Level } 3\end{array}$ \\
Level 4 & $\begin{array}{l}\text { Special polar products: Maps of permanent shadow, illumination levels, } \\
\text { surface and subsurface temperatures }\end{array}$ \\
\hline
\end{tabular}

within 6 months of acquisition. Level 2, 3 and 4 data products will be archived after the end of the one-year LRO mapping mission. Diviner's archived data products will be in the form of ASCII text files.

\subsection{Calibrated Radiances}

Diviner's Level 1 calibrated radiance product will be the starting point for the creation of all higher-level data products. Diviner's calibration approach is similar to that employed by MCS.

Diviner radiance measurements in 42 visible and 147 infrared detector channels will be acquired in the form of raw signal counts once every $128 \mathrm{msec}$. Raw counts are converted to calibrated radiances using a two-point calibration procedure, based on views of space, instrument blackbody targets, and solar calibration targets. Space views provide good zero signal, or offset, calibration for all channels, whereas blackbody views provide an accurate radiance standard for the infrared channels, allowing gain to be calculated. For the visible channels, the reflectivity along the line of sight can be related directly to solar target reflectivity, which has been characterized as a function of solar elevation and azimuth angle during laboratory calibration.

For the infrared detector channels, space and blackbody views are obtained at approximately ten equally-spaced times per orbit, and are used to calculate signal offset and gain. Interpolation between these discrete calibration points will be done using a simple polynomial fit. Signal counts for each channel are then converted linearly to a preliminary radiance by interpolating offsets and gains between calibration times. Finally, a simple polynomial expression, derived from laboratory linearity measurements on the flight instrument, is used to convert preliminary to calibrated radiance. A similar approach is used for the visible channels. Again space views are available ten times per orbit, but the solar calibration target is observed only once or twice per orbit when the position of the Sun is favorable. Linearity measurements are not available for this channel, but its radiometric accuracy and precision requirements are met by assuming a linear response. Both gain and offset are influenced by instrument temperature variations around the LRO orbit, and the calibration approach is designed to correct for this, in addition to longer term drifts. 

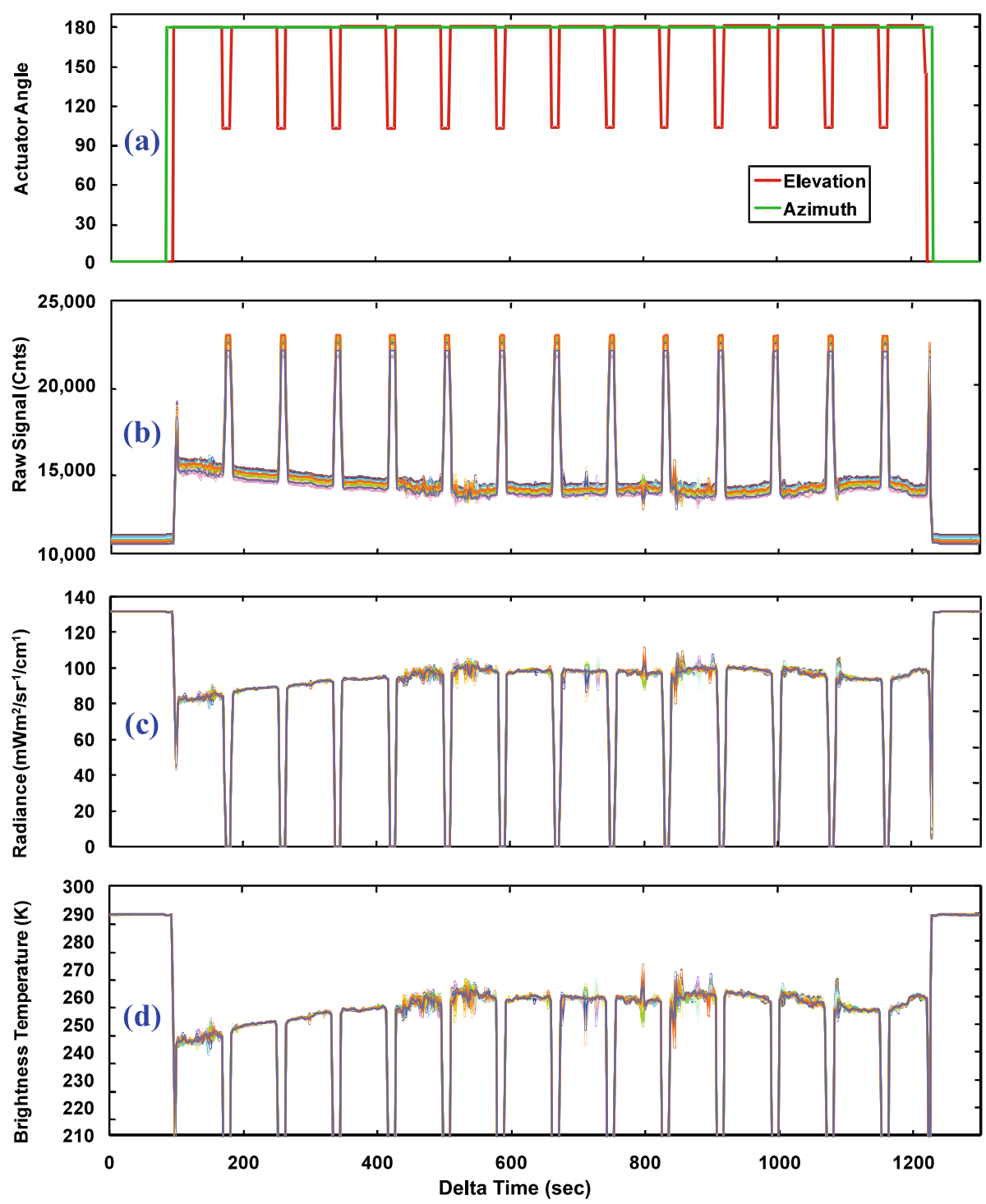

Fig. 37 Raw and calibrated MRO MCS 21.6 $\mu \mathrm{m}$ channel observations of the surface of Mars acquired in nadir pushbroom mapping mode. The data shown here were acquired during a 20 minute period in November, 2006 along a single MRO orbit track along $\sim 150^{\circ}$ east longitude extending from $8.3^{\circ} \mathrm{S}$ to $50.9^{\circ} \mathrm{N}$ latitude at approximately 15.25 hours Mars local time. Panel (a) displays instrument actuator azimuth (green) and elevation (red) angles. Elevation angles of $0^{\circ}, 103^{\circ}$ and $180^{\circ}$ correspond respectively to blackbody, space and nadir views. Panel (b) shows uncalibrated signals in counts for the 21 detectors of the MCS $21.6 \mu \mathrm{m}$ micron channel. The contrasting blackbody, space, and on-planet signals are evident, and the shifting of the curves relative to each other results from the different offsets and gains associated with each detector before calibration. Panel (c) shows calibrated radiances for the same time period and detector channels. The curves are now superimposed except for surface temperature structure seen in some of the nadir views. Panel (d) shows brightness temperatures corresponding to the radiances of panel (c). Martian surface temperatures vary over the range $240-270 \mathrm{~K}$ in this time period. Contrast across the detector arrays is caused primarily by solar illumination angle. Flat, featureless terrain produces very little contrast 
The calibration procedure that Diviner will employ for the Moon is similar to that illustrated in Fig. 37, which shows actuator angles, raw counts, radiances and brightness temperatures acquired by MCS for the surface of Mars in its $21.7 \mu \mathrm{m}$ channel.

\subsection{Higher-Level Data Products}

Diviner's Level 2 data product is a spatially and temporally averaged version of the global calibrated dataset for mapping and correlation with mapped data products provided by other instruments. The Level 3 and 4 data products are created by fitting the Diviner data with the results of models for the thermal behavior of the lunar surface and subsurface, the spectral emission and solar reflectance of the lunar surface, and maps of the Moon's topography and roughness. The approaches that will be employed to derive the Level 3 and 4 products will be described in detail in future publications.

\section{Summary}

The Diviner lunar radiometer experiment will provide comprehensive and rich global datasets that will support the exploration and science goals of the LRO mission. The instrument will be the first to systematically map the thermal emission of the lunar surface over the complete range of latitude, longitude, local time and season. The results of the investigation will be applicable to better defining and understanding the lunar thermal environment, the geologic history and composition of the lunar surface, and the distribution and history of lunar volatiles.

Acknowledgements We thank the following individuals for their contributions to the Diviner experiment: Henry Awaya and Nick Emis—Thermal Design, John Bousman-Mechanical Integration, Scott NolteElectronics and Integration support, Bradley Drake-Electronics assembly, Robert Hughes-Thermal Analysis, Peter Barry, Kim Plourde-Mission Assurance, Nick Taylor-Reliability, James Aragon-Quality Assurance, Robert Stephenson-Structures, Mike O'Connell-Dynamics, Todd Newell—Environmental Requirements, Valsamual Galaske-Mechanical Design, Paul Willis-Materials, Yuri Beregovski-Optics, Orland Harrison, Lee Wigglesworth-Electrical Ground Test Equipment, Albert Chen-Electronics, Cami Vongsouthy—Safety, Kelly Stanford-Electronic Parts, Tom Pierce—Electrical Compatibility, John Diehl— Ground Data Systems, Glenn Aveni-Contamination Control, Joyce Grunwald-Contract Manager, Laurie Guay-budgeting, Tarek Baayon and Amy Liu—Scheduling, Paul Cate-Procurement Support, Jon Temple - Design and Manufacture, Jason Perry-Filter Frame Development, Gary Hawkins and Richard Sherwood-Multilayer Filters, Peter Ade and Carol Tucker-Mesh Filters, Stewart Biggar-Goniometry, Mike Jakobson-Spectrophotometry, Paul Hayne, Michaela Shopland, Matt Siegler, Tim Tran and Joann Zhang-Thermal Vacuum Testing Support, Young Park and Thomas Luchik-Management Support, Arlin Bartels-Payload Management. The research described in this paper carried out at the Jet Propulsion Laboratory, California Institute of Technology, was done so under a contract with the National Aeronautics and Space Administration.

Open Access This article is distributed under the terms of the Creative Commons Attribution Noncommercial License which permits any noncommercial use, distribution, and reproduction in any medium, provided the original author(s) and source are credited.

\section{References}

C.J. Cremers, R.C. Birkebak, in Proc. Lunar Sci. Conf. 2nd (1971), pp. 2311-2315

M.B. Duke, L.R. Gaddis, G.J. Taylor, H.H. Schmitt, Rev. Mineral. Geochem. 60, 597-655 (2006)

W.C. Feldman, S. Maurice, D.J. Lawrence, R.C. Little, S.L. Lawson, O. Gasnault, R.C. Wiens, B.L. Barraclough, R.C. Elphic, T.H. Prettyman, J.T. Steinberg, A.B. Binder, J. Geophys. Res. 106, 23,231-23,251 (2001) 
B.T. Greenhagen, D.A. Paige, Lunar Planet. Sci. 37 (2006), abstract 2406

B.T. Greenhagen, D.A. Paige, Lunar Planet. Sci. 40 (2009), abstract 2255

R.R. Hodges Jr., in Proc. Lunar Planet. Sci. Conf. 11th (1980), pp. 2463-2477

J.C. Jaeger, A.F.A. Harper, Nature 166, 1026 (1950)

S.J. Keihm, M.G. Langseth Jr., in Proc. Lunar Sci. Conf. 4th (1973), pp. 2503-2513

S.L. Lawson, B.M. Jakosky, H.S. Park, Lunar Planet. Sci. 28 (1997), abstract 1375

S.L. Lawson, B.M. Jakosky, H.S. Park, M.T. Mellon, J. Geophys. Res. 105, 4273-4290 (2000)

L.M. Logan, G.R. Hunt, J.W. Salisbury, S.R. Balsamo, J. Geophys. Res. 78, 4983-5003 (1973)

D.J. McCleese, J.T. Schofield, R. Zurek, J.V. Martonchik, R.D. Haskins, D.A. Paige, R.A. West, D.J. Diner, J.R. Locke, M.F. Chrisp, W. Willis, F.W. Taylor, Appl. Opt. 25(23), 4232-4245 (1986)

D.J. McCleese R.D. Haskins, J.T. Schofield, R.W. Zurek, C.B. Leovy, D.A. Paige, F.W. Taylor, J. Geophys. Res. 97, 7735-7757 (1992)

D.J. McCleese, J.T. Schofield, F.W. Taylor, S.B. Calcutt, M.C. Foote, D.M. Kass, C.B. Leovy, D.A. Paige, P.L. Read, R.W. Zurek, J. Geophys. Res. 112, E05S06 (2007). doi:10.1029/2006JE002790

W.W. Mendell, Ph.D. Thesis. Rice University, Houston, TX, 1976

W.W. Mendell, F.J. Low, Moon 9, 97-103 (1974)

I. Mitrofanov, Space Sci. Rev. (2009, this issue)

F.H. Murcray, D.G. Murcray, W.J. Williams, J. Geophys. Res. 75, 2662-2669 (1970)

B.C. Murray, M.J. Wildey, Astrophys. J. 139, 734-750 (1964)

T.J. Nightingale, J. Crawford, Metrologia 28, 233-237 (1991)

A.M. Ono, Ph.D. Thesis. University of California, Los Angeles, CA (1999)

E. Pettit, S.B. Nicholson, Astrophys. J. 71, 102-135 (1930)

J.W. Salisbury, R.K. Vincent, L.M. Logan, G.R. Hunt, J. Geophys. Res. 75, 2671-2682 (1970)

J.W. Salisbury, G.R. Hunt, L.M. Logan, in Lunar Planet. Sci. Conf. 4th (1973), pp. 3191-3196

L.D. Stimpson, J.W. Lucas, J. Spacecr. Rockets 7, 1317-1322 (1970)

A.R. Vasavada, D.A. Paige, S.E. Wood, Icarus 141, 179-193 (1999)

K. Watson, B.C. Murray, H. Brown, J. Geophys. Res. 66, 3033-3045 (1961) 